\title{
Combinatorial Auctions Do Need Modest Interaction
}

\author{
SEPEHR ASSADI, Rutgers University, NJ, USA
}

\begin{abstract}
We study the necessity of interaction for obtaining efficient allocations in combinatorial auctions with subadditive bidders. This problem was originally introduced by Dobzinski, Nisan, and Oren (STOC'14) as the following simple market scenario: $m$ items are to be allocated among $n$ bidders in a distributed setting where bidders valuations are private and hence communication is needed to obtain an efficient allocation. The communication happens in rounds: In each round, each bidder, simultaneously with others, broadcasts a message to all parties involved. At the end, the central planner computes an allocation solely based on the communicated messages. Dobzinski et al. showed that (at least some) interaction is necessary for obtaining even an approximately efficient allocation: No non-interactive (1-round) protocol with polynomial communication (in the number of items and bidders) can achieve approximation ratio better than $\Omega\left(m^{1 / 4}\right)$, while for any $r \geq 1$, there exists $r$-round protocols that achieve $\widetilde{O}\left(r \cdot m^{1 /(r+1)}\right)$ approximation with polynomial communication. This in particular implies that $O(\log m)$ rounds of interaction suffice to obtain an approximately efficient allocation-namely, a polylog(m)-approximation.

A natural question at this point is to identify the "right" level of interaction (i.e., number of rounds) necessary to obtain approximately efficient allocations. In this article, we resolve this question by providing an almost tight round-approximation tradeoff for this problem: We show that for any $r \geq 1$, any $r$-round protocol that uses poly $(m, n)$ bits of communication can only approximate the social welfare up to a factor of $\Omega\left(\frac{1}{r} \cdot m^{1 /(2 r+1)}\right)$. This in particular implies that $\Omega\left(\frac{\log m}{\log \log m}\right)$ rounds of interaction are necessary for obtaining any allocation with a reasonable welfare approximation-namely, a constant or even a polylog $(m)$ approximation. Our work builds on the multi-party round-elimination technique of Alon, Nisan, Raz, and Weinstein (FOCS'15) - used to prove similar-in-spirit lower bounds for round-approximation tradeoff in unitdemand markets-and settles an open question posed initially by Dobzinski et al. (STOC'14) and subsequently by Alon et al. (FOCS'15).
\end{abstract}

CCS Concepts: - Theory of computation $\rightarrow$ Algorithmic mechanism design; Communication complexity;

Additional Key Words and Phrases: Combinatorial auctions, multi-party communication complexity, roundapproximation tradeoff

\section{ACM Reference format:}

Sepehr Assadi. 2020. Combinatorial Auctions Do Need Modest Interaction. ACM Trans. Econ. Comput. 8, 1, Article 3 (March 2020), 23 pages.

https://doi.org/10.1145/3381521

\footnotetext{
Work done while the author was a graduate student at University of Pennsylvania and was supported in part by National Science Foundation grants CCF-1552909, CCF-1617851, and IIS-1447470.

Author's address: S. Assadi, Princeton University, Princeton, NJ, USA; email: sassadi@cis.upenn.edu.

Permission to make digital or hard copies of all or part of this work for personal or classroom use is granted without fee provided that copies are not made or distributed for profit or commercial advantage and that copies bear this notice and the full citation on the first page. Copyrights for components of this work owned by others than ACM must be honored. Abstracting with credit is permitted. To copy otherwise, or republish, to post on servers or to redistribute to lists, requires prior specific permission and/or a fee. Request permissions from permissions@acm.org. (C) 2020 Association for Computing Machinery.

2167-8375/2020/03-ART3 \$15.00

https://doi.org/10.1145/3381521
} 


\section{INTRODUCTION}

In a combinatorial auction, we have a set $M$ of $m$ items and a set $N$ of $n$ bidders (or players ${ }^{1}$ ) with valuation functions $v_{i}: 2^{M} \rightarrow \mathbb{R}_{+}$. The goal is to find a collection of disjoint bundles $A_{1}, \ldots, A_{n}$ of items in $M$ (an allocation), that maximizes the social welfare defined as the sum of bidder's valuations for the allocated bundles, i.e., $\sum_{i \in N} v_{i}\left(A_{i}\right)$. We study the tradeoff between the amount of interaction between the bidders and the efficiency of the allocation in combinatorial auctions.

In our model, each bidder $i \in N$ only knows his or her valuation function $v_{i}$ and, hence, the bidders need to communicate to obtain even an approximately efficient allocation. Communication happens in rounds. In each round, each bidder $i$, simultaneously with others, broadcasts a message to all parties involved based on the valuation function $v_{i}$ and messages in previous rounds. In the last round, the central planner outputs the allocation solely based on the communicated messages. Notice that a "trivial solution" in this setting is for all players to communicate their entire input to the central planner who can then compute an efficient allocation; however, such a protocol is clearly infeasible in most settings, as it has an enormous communication cost. As such, we are interested in protocols with significantly less communication cost-typically exponentially smaller than the input size (polynomial in $m$ and $n$ )-that are able to compute allocations with a reasonable welfare approximation-namely, a constant or even a polylog $(m)$-approximation.

This model was first introduced by Dobzinski, Nisan, and Oren [13] to address the following fundamental question in economics:

"To what extent is interaction between individuals required in order to efficiently allocate resources between themselves?"

Dobzinski et al. considered this problem for two different classes of valuation functions: unitdemand valuations and subadditive valuations (see Section 2.1 for definition). For both settings, they showed that (at least some) interaction is necessary to obtain an efficient allocation: noninteractive (a.k.a. 1-round or simultaneous) protocols have enormous communication cost compared to interactive ones, while even allowing a modest amount of interaction allows for finding an approximately efficient allocation. We now elaborate more on these results.

For the case of matching markets with $n$ unit-demand bidders and $n$ items (and hence input-size of $n$ bits per player), Dobzinski et al. [13] proved a lower bound of $\Omega(\sqrt{n})$ on the approximation ratio of any simultaneous protocol that communicates $n^{o(1)}$ bits per each bidder. However, they showed that for any $r \geq 1$, there exists an $r$-round protocol that achieves an $O\left(n^{1 /(r+1)}\right)$ approximation by sending $O(\log n)$ bits per each bidder in each round. For the more general setting of combinatorial auctions with $n$ subadditive bidders and $m$ items (and hence input-size of $\exp (m)$ bits per player), they showed that the best approximation ratio achievable by simultaneous protocols with poly $(m, n)$ communication is $\Omega\left(m^{1 / 4}\right)$, while for any $r \geq 1$, there exists $r$-round protocols that achieve an approximation ratio of $\widetilde{O}\left(r \cdot m^{1 /(r+1)}\right)$. These results imply that in such markets, logarithmically many rounds of interaction in the market size suffice to obtain an approximately efficient allocation-namely, a polylog $(m)$-approximation.

A natural question left open by Dobzinski et al. [13] was to identify the amount of interaction necessary to obtain an efficient allocation in these markets. Recently, Alon, Nisan, Raz, and Weinstein [2] provided a partial answer to this question for matching markets: For any $r \geq 1$, any $r$-round protocol for unit-demand bidders in which each bidder sends at most $n^{o(1)}$ bits in each round can only achieve an $\Omega\left(n^{1 / 5^{r+1}}\right)$ approximation [2]. This implies that at least $\Omega(\log \log n)$ rounds of interaction are necessary to achieve an approximately efficient allocation in matching

\footnotetext{
${ }^{1}$ Throughout the article, we use the terms "bidder" and "player" interchangeably.
} 
markets. Alon et al. [2] further conjectured that the "correct" lower bound for the convergence rate in this setting is $\Omega(\log n)$; in other words, $\Omega(\log n)$ rounds of interaction are necessary for achieving an approximately efficient allocation.

Despite this progress for matching markets, the best-known lower bounds for the more general setup of combinatorial auctions with subadditive bidders remained the aforementioned 1-round lower bound of Reference [13], and a $(2-\varepsilon)$-approximation (for every constant $\varepsilon>0$ ) for any polynomial communication protocol with unrestricted number of rounds [14]. Indeed, obtaining better lower bounds for $r$-round protocols was posed as an open problem by Alon et al. [2], who also mentioned that: "from a communication complexity perspective, lower bounds in this setup are more compelling, since player valuations require exponentially many bits to encode, hence interaction has the potential to reduce the overall communication from exponential to polynomial."

\subsection{Our Results and Techniques}

In this article, we resolve the aforementioned open question of Dobzinski et al. [13] and Alon et al. [2] by proving an almost tight round-approximation tradeoff for polynomial communication protocols in subadditive combinatorial auctions.

MAIN RESULT. For any $r \geq 1$, any $r$-round protocol (deterministic or randomized) for combinatorial auctions with $n$ subadditive bidders and $m$ items that uses polynomial communication in $m$ and $n$ can only achieve an approximation ratio of $\Omega\left(\frac{1}{r} \cdot m^{1 / \Theta(r)}\right)$ to the social welfare.

We remark that this lower bound holds even when the valuations are XOS functions, a strict subclass of subadditive valuations (see Section 2.1 for definition).

Our main result, combined with the upper bound result of Reference [13], provides a nearcomplete understanding of the power of each additional round in improving the quality of the allocation in subadditive combinatorial auctions. Moreover, an immediate corollary of our result is that in these markets, $\Omega\left(\frac{\log m}{\log \log m}\right)$ rounds of interaction are necessary to achieve any allocation with a reasonable welfare approximation-namely, a constant or even a polylog $(m)$-approximation, which is tight up to an $O(\log \log m)$ factor. The qualitative message of this theoretical result is clear: A modest amount of interaction between individuals in a market is crucial for obtaining an efficient allocation.

Our Techniques. Our first step in establishing this result is proving a new lower bound for simultaneous (1-round) protocols. We deviate from Reference [13] by considering the problem of estimating the value of social welfare as opposed to finding the actual allocation; this problem can only be harder in terms of proving a lower bound, as any protocol that can find an approximate allocation can also be used to estimate the value of social welfare with one additional round and $O(n)$ additional communication using a trivial reduction (see Section 2.2). As a result, the kind of combinatorial arguments used in Reference [13] seem not sufficient for our purpose, and we instead prove our lower bound using information-theoretic machinery and in particular a directsum style argument. This counterintuitive switch to establishing a lower bound for a seemingly harder problem, however, leads to a more modular proof that allows us to further carry out our results to multi-round protocols.

The proof of our multi-round lower bound is through an inductive argument generally referred to as round-elimination in the communication complexity literature. On a high level, a roundelimination argument works as follows: Given an $r$-round protocol that uses "too little communication," one constructs an $(r-1)$-round protocol on "simpler instances" (in some carefully chosen measure) by embedding the smaller instance in the larger one and essentially ignoring the first 
message of the $r$-round protocol (as it is somewhat "irrelevant" for solving these simpler instances) and running the protocol from its second round onwards. By a careful argument, one then shows that the resulting protocol can solve the simpler $(r-1)$-round instances with too little communication in $r-1$ rounds, which contradicts the induction hypothesis, hence implying the desired lower bound on communication cost of $r$-round protocols.

We implement this strategy by following the multi-party round-elimination technique of Alon et al. [2]. We create a recursive family of hard distributions $\mathcal{D}_{1}, \mathcal{D}_{2}, \ldots$ whereby for any $r \geq 1, \mathcal{D}_{r}$ is the hard input distribution for $r$-round protocols. Each instance in $\mathcal{D}_{r}$ is a careful combination of exponentially many sub-instances sampled from $\mathcal{D}_{r-1}$. One of these sub-instances is "special" in that to solve the original instance, the players also need to solve this special sub-instance completely. However, the players are not able to identify this special sub-instance locally and hence need to spend one round of interaction only for this purpose. In other words, we prove that the first round of protocol does not convey much information about the special instance beyond its identity. Using a further round-elimination argument, we inductively show that, since solving the special instance is hard for $(r-1)$-round protocols, solving the original instance should be hard for $r$-round protocols as well.

Similar to Reference [2], and unlike typical two-player round-elimination arguments (see, e.g., References [28, 32]), eliminating a round in our round-elimination argument requires a reduction from "low dimensional" instances (with fewer players and items) to "high dimensional" instances. This reduction is delicate, as the players need to "complete" their inputs in the higher dimensional instance by independently sampling the "missing part" conditioned on the first message of the protocol without any further communication, while this distribution is a correlated distribution.

Furthermore, in contrast to Reference [2], our sub-instances in each distribution are overlapping (as otherwise exponentially many sub-instances cannot be embedded inside a single polynomially larger instance) and hence may interfere with each other, potentially diminishing the role of the special instance. We overcome this obstacle by embedding these sub-instances based on a family of small-intersection sets to limit the potential overlap between the sub-instances and prove that solving the special instance is crucial even in the presence of these overlaps. It is worth pointing out that this approach allows us to avoid the doubly-exponential rate of growth in the size of instances across different rounds in Reference [2], resulting in exponentially better dependence on the parameter $r$ in our lower bound compared to Reference [2]. Finally, since our lower bound is for estimating the value of social welfare (as opposed to finding an allocation), we need a different embedding argument in our reduction than the one used in Reference [2]. ${ }^{2}$ In particular, we now embed the low-dimensional instance in multiple places of the high-dimensional instance as opposed to only one. We elaborate more on details of the lower bound in Sections 3 and 4.

\subsection{Further Related Work}

The communication complexity of combinatorial auctions has received quite a lot of attention in the literature. It is known that for arbitrary valuations, exponential amount of communication is needed to obtain an $\left(m^{1 / 2-\varepsilon}\right)$-approximate allocation (for every constant $\varepsilon>0$ ) [30] (see also Reference [29]), and this is also tight [1, 10, 25, 27]. For subadditive valuations, a constant factor approximation to the social welfare can be achieved in our model using only polynomial communication [14, 15, 19-21, 26, 33] (and polynomially many rounds of interaction); in particular, Feige [20] developed a 2-approximation polynomial communication protocol for this problem and

\footnotetext{
${ }^{2}$ Our problem corresponds to the problem of estimating the size of a maximum matching as opposed to finding an approximate matching in the setting of Reference [2]. To the best of our knowledge, no non-trivial lower bounds are known for the matching size estimation problem in the setting of Reference [2]; see Reference [5] for further details.
} 
Dobzinski, Nisan, and Schapira [14] proved that obtaining $(2-\varepsilon)$-approximation (for any constant $\varepsilon>0$ ) requires exponential communication (regardless of the number of rounds). Moreover, Dütting and Kesselheim [19] designed an $O(\log m)$-approximation protocol with polynomial communication for subadditive combinatorial auctions in which each bidder needs to communicate exactly once; however, this protocol still requires $n$ rounds of interaction in our model, as the players need to communicate in a round-robin fashion, making the message sent by a bidder crucially depending on the messages communicated earlier by the previous bidders.

Another line of relevant research considers the case where the valuation of the bidders is chosen independently from a commonly known distribution (see, e.g., References [18, 22, 23]) and aims to design "simple" and simultaneous protocols that achieve an approximately efficient allocation. The main difference between this setting and ours is that we are interested in arbitrary distributions of inputs for the bidders that are not necessarily product distributions; as already shown by the strong impossibility results of Reference [13], the aforementioned type of protocols cannot provably exist in our model when input distributions are correlated. Finally, we point out that "incompressability" results are also known for subadditive valuations: Any polynomial-length encoding of subadditive valuations must lose $\Omega(\sqrt{m})$ in precision [6, 7].

We refer the interested reader to Reference [13] for a comprehensive summary of related work and further discussion on the role of interaction in markets.

\subsection{Subsequent Work}

Subsequent to the conference version of this article [3], Braverman and Oshman [9] improved the lower bounds of Alon et al. [2] for unit-demand markets to achieve an almost tight bound on the round complexity of this problem. In particular, they proved that $\Omega\left(\frac{\log n}{\log \log n}\right)$ rounds of interaction are necessary to achieve an approximately efficient allocation in unit-demand (matching) markets. While this result is qualitatively similar to our result in this article for (subadditive) combinatorial auctions, our result and that of Reference [9] are incomparable in that neither implies or strengthens the other in any way. We also point out that in terms of techniques, our article and Reference [9] are entirely disjoint.

Furthermore, Assadi and Khanna [4] generalized the techniques in this article and developed a framework for proving communication complexity lower bounds for bounded-round protocols. This framework was in particular used to settle the round complexity of distributed maximum coverage problem in a communication setting similar to the one considered in this article. We refer the interested reader to Reference [4] for more details.

\section{PRELIMINARIES}

Notation. For any integer $a \geq 1$, we let $[a]:=\{1, \ldots, a\}$. We say that a set $S \subseteq[n]$ with $|S|=s$ is a $s$-subset of $[n]$. For a $k$-dimensional tuple $X=\left(X_{1}, \ldots, X_{k}\right)$ and index $i \in[k]$, we define $X^{<i}:=\left(X_{1}, \ldots, X_{i-1}\right)$ and $X^{-i}:=\left(X_{1}, \ldots, X_{i-1}, X_{i+1}, \ldots, X_{k}\right)$. We use capital letters to denote random variables. For a random variable $A, \operatorname{supp}(A) \operatorname{denotes~the~support~of~} A$ and $\operatorname{dist}(A) \operatorname{denotes}$ its distribution. We further define $|A|:=\log |\operatorname{supp}(A)|$. We write $A \perp B \mid C$ to denote that the random variables $A$ and $B$ are independent conditioned on $C$. We use "w.p." to mean "with probability."

Concentration bounds. Throughout, we use the following version of the Chernoff bound for negatively correlated random variables first proved by Reference [31]; see also References [17, 24].

Proposition 2.1 (Chernoff Bound). Let $X_{1}, \ldots, X_{n}$ be negatively correlated random variables taking values in $[0,1]$ and let $X:=\sum_{i=1}^{n} X_{i}$. Then, for any $\alpha \geq 2 e^{2}$,

$$
\operatorname{Pr}(X \geq \alpha \cdot \mathbb{E}[X]) \leq \exp (-\Omega(\alpha \cdot \mathbb{E}[X])) .
$$


Intersecting families. The following combinatorial construction plays a crucial role in our proofs:

Definition 1. A $(p, q, t, \ell)$-intersecting family $\mathcal{F}$ is a collection of $p$ subsets of $[q]$ each of size $t$, such that for any two distinct sets $S, T \in \mathcal{F},|S \cap T| \leq \ell$.

We prove the existence of an exponentially large intersecting family with a small pair-wise intersection using a probabilistic argument. The proof of this lemma is standard (see, e.g., Reference [29] for a similar statement) and is provided here for completeness.

LEMMA 2.2. For any integer $r \geq 1$, any parameter $\varepsilon>0$, and any integer $k \geq\left(2 e^{2} \cdot r^{2}\right)^{\frac{1}{\varepsilon}}$, there exists $a(p, q, t, \ell)$-intersecting family with $p=\exp \left(\Theta\left(k^{2 r-2+\varepsilon}\right)\right), q=k^{2 r}+r \cdot k^{2 r-1}, t=r \cdot k^{2 r-1}$, and $\ell=k^{2 r-2+\varepsilon}$.

Proof. Let $\mathcal{F}$ be a family of $p$ sets (for $p$ to be determined later), each chosen independently and uniformly at random from all $t$-subsets of $[q]$. Fix any pair of sets $S, T \in \mathcal{F}$; for each element $a \in S$, define the random variable $X_{a} \in\{0,1\}$ that is 1 iff $a \in T$ also. We have $\mathbb{E}\left[X_{a}\right] \leq r / k$. Let $X=$ $\sum_{a \in S} X_{a}$ denote $|S \cap T|$; hence, $\mathbb{E}[X] \leq r^{2} \cdot k^{2 r-2}$. Since $X_{a}$ 's are negatively correlated random variables, by Chernoff bound (Proposition 2.1 with $\alpha \geq k^{\varepsilon} / r^{2} \geq 2 e^{2}$ by lemma statement),

$$
\operatorname{Pr}(|S \cap T|>\ell)=\operatorname{Pr}\left(X>k^{2 r-2+\varepsilon}\right)=\operatorname{Pr}\left(X>k^{\varepsilon} / r^{2} \cdot \mathbb{E}[X]\right) \leq \exp \left(-\Omega\left(k^{2 r-2+\varepsilon}\right)\right) .
$$

By a union bound over all possible choices for $S, T \in \mathcal{F}$,

$$
\operatorname{Pr}(\exists S, T \in \mathcal{F}:|S \cap T|>\ell) \leq \sum_{S \neq T \in \mathcal{F}} \operatorname{Pr}(|S \cap T|>\ell) \leq\left(\begin{array}{l}
p \\
2
\end{array}\right) \cdot \exp \left(-\Omega\left(k^{2 r-2+\varepsilon}\right)\right) .
$$

Taking $p=\exp \left(\Theta\left(k^{2 r-2+\varepsilon}\right)\right)$ ensures that with some non-zero probability, the set $\mathcal{F}$ is a $(p, q, t, \ell)$ intersecting family, implying the existence of such a family.

\subsection{Combinatorial Auctions}

We have a set $N$ of $n$ bidders and a set $M$ of $m$ items. Each bidder $i \in N$ has a valuation function $v_{i}: 2^{M} \rightarrow \mathbb{R}_{+}$, which assigns a value to each bundle of items. We assume that $v_{i}(\emptyset)=0$ and $v_{i}(\cdot)$ is non-decreasing. The goal is to maximize the social welfare defined as $\max _{\left(A_{1}, \ldots, A_{n}\right)} \sum_{i \in N} v_{i}\left(A_{i}\right)$, where $\left(A_{1}, \ldots, A_{n}\right)$ ranges over all possible allocations of items in $M$ to bidders in $N$ such that bidder $i$ receives the bundle $A_{i}$.

A valuation function $v(\cdot)$ is subadditive iff for any two bundles of items $S, T \subseteq M, v(S \cup T) \leq$ $v(S)+v(T)$. A valuation function is additive iff for any bundle $S \subseteq M, v(S)=\sum_{j \in S} v(\{j\})$. A valuation function is XOS iff there exists $r$ additive valuation functions $a_{1}, \ldots, a_{r}$ such that for all bundles $S \subseteq M, v(S)=\max _{r} a_{r}(S)$. Each function $a_{j}$ is called a clause of $v$ and for any bundle $S$, any clause $a \in \arg \max _{r} a_{r}(S)$ is referred to as a maximizing clause of $S$. Finally, a valuation function is unit-demand iff for any $S \subseteq M, v(S)=\max _{j \in S} v(\{j\})$. It is easy to verify that both XOS and unit-demand functions are also subadditive.

Notice that, in general, subadditive and XOS valuation functions require $\exp (m)$ many bits for representation, while unit-demand valuation functions can be represented with $O(m)$ numbers, i.e., by describing the value of each singleton set. As such, in subadditive combinatorial auctions, we are interested in protocols that can reduce the communication from exponential in $m$ to polynomial, while in unit-demand auctions, we mainly seek protocols that reduce the communication from linear in $m$ to logarithmic.

\subsection{Communication Model}

We use the (number-in-hand) multiparty communication model with shared blackboard: There are $n$ players (corresponding to the bidders) receiving inputs $\left(x_{1}, \ldots, x_{n}\right)$, jointly distributed according 
to a prior distribution $\mathcal{D}$ on $\mathcal{X}_{1} \times \cdots \times \mathcal{X}_{n}$. The communication proceeds in rounds where in each round $r$, the players simultaneously write a message on a shared blackboard visible to all parties. In a deterministic protocol, the message sent by any player $i$ in each round can only depend on the private input of the player, i.e., $x_{i}$, plus the messages of all players in previous rounds, i.e., the content of the blackboard. In a randomized protocol, we further allow the players to have access to both public and private randomness and require that the output be correct with some constant probability more than half-say, $2 / 3$ (see below for more details).

For a protocol $\pi$, we use $\Pi=\left(\Pi_{1}, \ldots, \Pi_{n}\right)$ to denote the transcript of the message communicated by the players (i.e., the content of the blackboard). In addition to the $n$ players, there exists also an $(n+1)$-th party called the referee that does not have any input and is responsible for outputting the answer in the last round solely based on content of the blackboard $\Pi$ (plus the public randomness in case of randomized protocols). Finally, the communication cost of the protocol $\pi$, denoted by $\|\pi\|$, is the sum of worst-case length of the messages communicated by all players, i.e., $\|\pi\|=\sum_{i=1}^{n}\left|\Pi_{i}\right|$. We emphasize that for randomized protocols the communication cost is taken to be the worst-case length of the messages over both randomness of the distribution and the protocol. We note that, in this model, we are only interested in the number of rounds and the communication cost of the protocols and, hence, we do not restrict the runtime of the algorithms used by the players and the referee. This clearly can only strengthen our lower bound.

Approximation guarantee. We consider protocols that are required to estimate the maximum value of social welfare in any instance $I$ of a combinatorial auction (denoted by sw $(I)$ ). More formally, a $\delta$-error $\alpha$-approximation protocol needs to, for each input instance $I$ sampled from $\mathcal{D}$, output a number in the range $\left[\frac{1}{\alpha} \cdot \operatorname{sw}(I), \operatorname{sw}(I)\right]$ w.p. at least $1-\delta$, where the randomness is over the distribution $\mathcal{D}$ (and the randomness of protocol in case of randomized protocols).

This problem is provably easier than finding an approximate allocation in the interactive setting: any $r$-round protocol for finding an approximate allocation can be used to obtain an $(r+1)$-round protocol for estimating the value of social welfare with $O(n)$ additional communication; simply compute the approximate allocation in the first $r$ rounds and spend one additional round in which each player declares her value for the assigned bundle to the referee. It was shown very recently in Reference [8] that this loss of one round in the reduction is unavoidable (see Section 3 for further details). However, this extra one round is essentially negligible for our purpose, as we are interested in the asymptotic dependence of the approximation ratio and the number of rounds.

\subsection{Tools from Information Theory}

We briefly review some basic definitions and facts from information theory that are used in this article. We refer the interested reader to the excellent text by Cover and Thomas [11] for an introduction to this field and the proofs of the claims in this section (see Chapter 2).

In the following, we denote the Shannon Entropy of a random variable $A$ by $\mathbb{H}(A)$ defined as $\mathbb{H}(A):=\mathbb{E}_{a \sim A}\left[\log \frac{1}{\operatorname{Pr}(A=a)}\right]$ and the mutual information of two random variables $A$ and $B$ by $\mathbb{I}(A ; B):=\mathbb{H}(A)-\mathbb{H}(A \mid B)=\mathbb{H}(B)-\mathbb{H}(B \mid A)$. We use $H_{2}(\cdot)$ to denote the binary entropy function where for any real number $0<\delta<1, H_{2}(\delta):=\delta \log \frac{1}{\delta}+(1-\delta) \log \frac{1}{1-\delta}$. The proof of the following basic properties of entropy and mutual information can be found in Reference [11], Chapter 2.

FACT 2.3. Let $A, B$, and $C$ be three (possibly correlated) random variables.

(1) $0 \leq \mathbb{H}(A) \leq|A|$ and $\mathbb{H}(A)=|A|$ iff $A$ is uniformly distributed over its support.

(2) $\mathbb{I}(A ; B \mid C) \geq 0$. The equality holds iff $A \perp B \mid C$.

(3) $\mathbb{H}(A \mid B, C) \leq \mathbb{H}(A \mid B)$. The equality holds iff $A \perp C \mid B$. 
(4) $\mathbb{I}(A, B ; C)=\mathbb{I}(A ; C)+\mathbb{I}(B ; C \mid A)$ (chain rule of mutual information).

(5) Suppose $f(A)$ is a deterministic function of $A$, then $\mathbb{I}(f(A) ; B \mid C) \leq \mathbb{I}(A ; B \mid C)$ (data processing inequality).

The following Fano's inequality states that if a random variable $A$ can be used to estimate the value of another random variable $B$, then $A$ should "consume" most of $B$ 's entropy.

FACT 2.4. Let $A, B$ be random variables and $f$ be a function that given $A$ predicts a value for $B$. Suppose $B$ is binary and $\operatorname{Pr}(f(A) \neq B) \leq \delta$, then $\mathbb{H}(B \mid A) \leq H_{2}(\delta)$.

We also use the following two simple propositions. The proofs are standard and are only provided for completeness.

Proposition 2.5. For variables $A, B, C, D$, if $A \perp D \mid C$, then, $\mathbb{I}(A ; B \mid C) \leq \mathbb{I}(A ; B \mid C, D)$.

Proof. Since $A$ and $D$ are independent conditioned on $C$, by Fact 2.3-(3), $\mathbb{H}(A \mid C)=\mathbb{H}(A \mid$ $C, D)$ and $\mathbb{H}(A \mid C, B) \geq \mathbb{H}(A \mid C, B, D)$. We have,

$$
\begin{aligned}
\mathbb{I}(A ; B \mid C) & =\mathbb{H}(A \mid C)-\mathbb{H}(A \mid C, B)=\mathbb{H}(A \mid C, D)-\mathbb{H}(A \mid C, B) \\
& \leq \mathbb{H}(A \mid C, D)-\mathbb{H}(A \mid C, B, D)=\mathbb{I}(A ; B \mid C, D) .
\end{aligned}
$$

Proposition 2.6. For variables $A, B, C, D$, if $A \perp D \mid B, C$, then, $\mathbb{I}(A ; B \mid C) \geq \mathbb{I}(A ; B \mid C, D)$.

Proof. Since $A \perp D \mid B, C$, by Fact 2.3-(3), $\mathbb{H}(A \mid B, C)=\mathbb{H}(A \mid B, C, D)$. Moreover, since conditioning can only reduce the entropy (again by Fact 2.3-(3)),

$$
\begin{aligned}
\mathbb{I}(A ; B \mid C) & =\mathbb{H}(A \mid C)-\mathbb{H}(A \mid B, C) \geq \mathbb{H}(A \mid D, C)-\mathbb{H}(A \mid B, C) \\
& =\mathbb{H}(A \mid D, C)-\mathbb{H}(A \mid B, C, D)=\mathbb{I}(A ; B \mid C, D) .
\end{aligned}
$$

For two distributions $\mu$ and $v$ over the same probability space, the Kullback-Leibler divergence between $\mu$ and $v$ is defined as $\mathbb{D}(\mu \| v):=\mathbb{E}_{a \sim \mu}\left[\log \frac{\operatorname{Pr}_{\mu}(a)}{\operatorname{Pr}_{v}(a)}\right]$. We have,

FACT 2.7. For random variables $A, B, C$,

$$
\mathbb{I}(A ; B \mid C)=\mathbb{E}_{(b, c) \sim \operatorname{dist}(B, C)}[\mathbb{D}(\operatorname{dist}(A \mid C=c) \| \operatorname{dist}(A \mid B=b, C=c))] .
$$

We denote the total variation distance between two distributions $\mu$ and $v$ over the same probability space $\Omega$ by $\|\mu-v\|=\frac{1}{2} \cdot \sum_{x \in \Omega}\left|\operatorname{Pr}_{\mu}(x)-\operatorname{Pr}_{v}(x)\right|$. The following Pinsker's inequality bounds the total variation distance between two distributions based on their KL-divergence,

FACt 2.8 (Pinsker's ineQuality). For any two distributions $\mu$ and $v,\|\mu-v\| \leq \sqrt{\frac{1}{2} \cdot \mathbb{D}(\mu \| v)}$.

Finally,

FACT 2.9. Suppose $\mu$ and $v$ are two distributions for an event $\mathcal{E}$, then, $\operatorname{Pr}_{\mu}(\mathcal{E}) \leq \operatorname{Pr}_{v}(\mathcal{E})+\|\mu-v\|$.

\section{WARMUP: A LOWER BOUND FOR SIMULTANEOUS PROTOCOLS}

Our main lower bound result is based on analyzing a recursive family of distributions. As a warmup, we analyze the base case of this recursive construction in this section and prove a lower bound for 1-round (simultaneous) protocols. Formally,

Theorem 2. For any sufficiently small constant $\varepsilon>0$, any simultaneous protocol (possibly randomized) for combinatorial auctions with subadditive (even XOS) bidders that can approximate the value of social welfare to a factor of $m^{\frac{1}{3}-\varepsilon}$ w.p. at least $2 / 3$ requires $\exp \left(m^{\Omega(\varepsilon)}\right)$ bits of communication in the worst case. 
Theorem 2 is proved in Section 3.2. It is worth mentioning that the bound established in Theorem 2 on the approximation ratio of simultaneous protocols is asymptotically tight: Dobzinski et al. [13] developed a simultaneous protocol that can approximate the social welfare up to an $\widetilde{O}\left(\mathrm{~m}^{1 / 3}\right)$ factor using only polynomial communication.

As pointed out earlier, Dobzinski et al. [13] have previously proved an $\Omega\left(m^{1 / 4}\right)$ lower bound on the approximation ratio of the protocols that can find an approximate allocation. We should remark that this lower bound of Reference [13] and our lower bound in Theorem 2 are incomparable in that neither imply (or strengthen) the other. The reason is that while the problem of estimating the social welfare is provably easier than the problem of finding an approximate allocation, the reduction requires one additional round of interaction and hence, in general, a simultaneous protocol for the problem of finding the allocation only implies a 2-round (and not a simultaneous) protocol for the social welfare estimation problem. ${ }^{3}$ Interestingly, for $n=2$ players, Braverman et al. [8] very recently showed that estimating the social welfare is indeed provably harder than finding an approximate allocation for simultaneous protocols. In the light of this result, it seems plausible that one can improve the protocol of Reference [13] and obtain an $O\left(\mathrm{~m}^{1 / 4}\right)$-approximation protocol for finding an approximate allocation (matching the lower bound of Reference [13]); however, Theorem 2 suggests that such a protocol should necessarily be oblivious to the welfare of the allocation it provides.

\subsection{A Hard Input Distribution for Simultaneous Protocols}

In this section, we propose a hard input distribution $\mathcal{D}_{1}$ for simultaneous protocols used in the proof of Theorem 2 and state several of its properties that are needed in the proof. We start by providing an informal description of the distribution $\mathcal{D}_{1}$.

Let $k$ be an integer and consider a set $N$ of $n=k^{2}$ players and $M$ of $m=k^{3}$ items. Each bidder $i \in N$ is given an exponentially large (in $k$ ) collection $\mathcal{F}_{i}$ of item-sets of size $k$ each, such that for all $S \subseteq M, v_{i}(S)=\max _{T \in \mathcal{F}_{i}}|S \cap T|$ (recall that the input to player $i$ is the valuation function $v_{i}(\cdot)$, which is an XOS-valuation function). Additionally, the sets in $\mathcal{F}_{i}$ are "barely overlapping," in the sense that for any two sets $S, T \in \mathcal{F}_{i},|S \cap T|<k^{\varepsilon}$ (for any constant $\varepsilon>0$ ).

This construction ensures that locally each player is confronted with exponentially many high value bundles (sets in $\mathcal{F}_{i}$ ) that look "exactly the same." However, these collections across different players are chosen in a correlated way such that except for a single "special bundle" $T_{j} \in \mathcal{F}_{i}$ (for each $i \in N$ ), the items in all other bundles are chosen (mostly) from a (relatively small) set of $k^{2}$ "shared" items across all players. The special bundles, however, consist of "unique" items. This implies that globally (i.e., when considering valuation functions of all players) each player has a special bundle, and these special bundles are crucial to obtaining any $k^{1-\varepsilon}$-approximate allocation (recall that $k^{1-\varepsilon}=m^{\frac{1-\varepsilon}{3}}$ ).

We then use an additional randomization trick to ensure that any instance sampled from $\mathcal{D}_{1}$ either has a "large" social welfare (w.p., say, half) or a "small" one (with the remaining probability): We drop some of the bundles from the collection $\mathcal{F}_{i}$ of each player $i \in N$ randomly (in a correlated way) to create two sub-distributions such that in one of them none of the special bundles are dropped and hence the social welfare is $k^{3}$, and in the other one all special bundles are dropped and hence the social welfare is at most $k^{2+\varepsilon}\left(k^{2}\right.$ for shared items plus $k^{\varepsilon}$ intersection from any other bundle (in $\mathcal{F}_{i}$ ) for each of the $k^{2}$ players). This randomization trick now requires the referee to figure out whether or not the special bundles are dropped, which we prove is a hard task, i.e.,

\footnotetext{
${ }^{3}$ Note, however, that the $\widetilde{O}\left(\mathrm{~m}^{1 / 3}\right)$-approximation protocol of Reference [13] can already compute the welfare of the allocated allocation and hence does not need an additional round for estimating the social welfare, implying the tightness of the bounds in Theorem 2 .
} 
requires a large communication. This completes the description of our hard distribution. We now formally define $\mathcal{D}_{1}$.

Distribution $\mathcal{D}_{1}(N, M)$. A hard input distribution for simultaneous protocols.

Input: Collections $N$ of $n=k^{2}$ players and $M$ of $m=2 k^{3}$ items.

Output: A set of $n$ valuation functions $\left(v_{1}, \ldots, v_{n}\right)$ for the players in $N$.

(1) Let $\mathcal{S}=\left\{S_{1}, \ldots, S_{p}\right\}$ be a $(p, q, t, \ell)$-intersecting family with $p=\exp \left(\Theta\left(k^{\varepsilon}\right)\right), q=k^{2}+k$, $t=k$, and $\ell=k^{\varepsilon}$ (guaranteed to exist by Lemma 2.2).

(2) Pick $j^{\star} \in[p]$ and $\theta \in\{0,1\}$ independently and uniformly at random.

(3) For each player $i \in N$ independently,

(a) Denote by $\mathcal{F}_{i}$ the private collection of player $i$ (used below to define the valuation function $v_{i}$ ), initialized to be a copy of $\mathcal{S}$ on the universe $[q]$.

(b) Let $x_{i} \in\{0,1\}^{p}$ be a $p$-dimensional vector whereby $x_{i}\left(j^{\star}\right)=\theta$ and for any $j \neq j^{\star}$, $x_{i}(j)$ is chosen uniformly at random from $\{0,1\}$.

(c) For any $j \in[p]$, if $x_{i}(j)=0$, remove the set $S_{j}$ from $\mathcal{F}_{i}$ and otherwise keep $S_{j}$ in $\mathcal{F}_{i}$.

(4) Pick a random permutation $\sigma$ of $M$. For the $i$ th player in $N$, map the $j$ th item in $[q] \backslash S_{j \star}$ to $\sigma(j)$. Moreover, map the $j$ th item in $S_{j^{\star}}$ to $\sigma\left(k^{2}+(i-1) \cdot k+j\right)$. Under this mapping, the private collection $\mathcal{F}_{i}$ of player $i$ consists of at most $p$ sets of $t=k$ items from $M$.

(5) For all $i \in N$, define the valuation function of player $i$ as $v_{i}(S)=\max _{T \in \mathcal{F}_{i}}|S \cap T|$.

We use $\mathcal{D}_{1}$ to denote the distribution $\mathcal{D}_{1}(N, M)$ whenever the sets $N$ and $M$ are clear from the context (or are irrelevant). We should note right away that from the description of distribution $\mathcal{D}_{1}$ it should be clear that having $m=k^{3}+k^{2}$ items also suffices for the construction (instead of $m=2 k^{3}$ used in $\mathcal{D}_{1}$ ); the extra items are added only to simplify the math. We make several observations about the distribution $\mathcal{D}_{1}$.

OBSERvation 3.1. The valuation function of each bidder $i \in[n]$ in the distribution $\mathcal{D}_{1}$ is an XOS valuation (and hence is also subadditive) whereby each set $T \in \mathcal{F}_{i}$ defines a clause in which all items in $T$ have value 1 and all other items have value 0.

For any player $i \in N$, we define the labeling function $\phi_{i}$ as the function used to map the items in $[q]$ to $M$. Notice that $\phi_{i}$ is a function of $\sigma$ and index $j^{\star}$.

ObSeRvation 3.2. The input to player $i$ can be uniquely identified by the pair $\left(x_{i}, \phi_{i}\right)$, as $x_{i}$ defines the private collection $\mathcal{F}_{i}$ over the items [q], and $\phi_{i}$ specifies the actual labeling of the items in $M$ in the instance.

We also point out a crucial property of this distribution: Each player $i \in N$ is oblivious to which of the sets $S_{j}$ (for $j \in[p]$ ) is the set $S_{j^{\star}}$. More formally,

Observation 3.3. Conditioned on the input $\left(x_{i}, \phi_{i}\right)$ to player $i$, the index $j^{\star} \in[p]$ is chosen uniformly at random.

Recall that for an instance $I$ of combinatorial auctions (and in particular $I \sim \mathcal{D}_{1}$ ), $\operatorname{sw}(I)$ denotes the maximum value of social welfare, i.e., $\operatorname{sw}(I):=\max _{\left(A_{1}, \ldots, A_{n}\right)} \sum_{i \in N} v_{i}\left(A_{i}\right)$, where $\left(A_{1}, \ldots, A_{n}\right)$ ranges over all possible allocation of items. The following lemma establishes a bound on the social welfare of any instance sampled from $\mathcal{D}_{1}$.

Lemma 3.4. For any $I \sim \mathcal{D}_{1}$, (i) if $\theta=1$, then $\operatorname{sw}(I)=k^{3}$, and (ii) if $\theta=0$, then $\operatorname{sw}(I) \leq 2 k^{2+\varepsilon}$.

Proof. Suppose first that $\theta=1$. In this case, $x_{i}\left(j^{\star}\right)=1$ for all bidders $i \in N$, implying that the set $S_{j^{\star}}$ is not removed from any private collection $\mathcal{F}_{i}$. Moreover, the mapping $\sigma$ maps the items 
in $S_{j \star}$ to a unique set of items and hence the allocation $\left(A_{1}, \ldots, A_{n}\right)$, where $A_{i}$ is the set of items $\sigma\left(k^{2}+(i-1) \cdot k+1\right) \ldots \sigma\left(k^{2}+i \cdot k\right)$, results in a welfare of $k^{3}$, which is clearly maximum.

Now consider the case $\theta=0$. In this case, $x_{i}\left(j^{\star}\right)=0$ for all bidders $i \in N$, and hence the set $S_{j^{\star}}$ is missing from all private collections. Recall that items in $[q] \backslash S_{j^{\star}}$ (across all players) are mapped to the first $k^{2}$ items of $M$ (according to the ordering $\sigma$ ). By the intersecting family property of the set $\mathcal{S}$, the intersection of $S_{j \star}$ with any other set in $\mathcal{S}$, and consequently, any other set in any $\mathcal{F}_{i}$ (for $i \in N$ ) is at most $\ell=k^{\varepsilon}$ items. This means that in any allocation, bidder $i$ can only "benefit" from at most $k^{\varepsilon}$ elements in $\sigma\left(k^{2}+1\right) \ldots \sigma\left(2 k^{3}\right)$. Consequently, in this case, $\operatorname{sw}(I)$ is at most $k^{2}$ (accounting for all the first $k^{2}$ items of $\sigma$ ) plus $k^{2+\varepsilon}$ (accounting for $k^{\varepsilon}$ benefit from each of the $k^{2}$ players).

We note that even though, by Lemma 3.4, the social welfare of instances sampled from $\mathcal{D}_{1}$ varies significantly based on the value of $\theta$, it is easy to see that the welfare maximizing allocation is almost the same regardless of the value of $\theta$. As such, our proof of Theorem 2 does not also imply a lower bound for the problem of finding approximate allocations.

\subsection{The Lower Bound for Distribution $\mathcal{D}_{1}$}

Let $\pi$ be a public coin simultaneous protocol that can output an $\left(m^{\frac{1-\varepsilon}{3}}\right)$-approximation to the social welfare of any instance $I \sim \mathcal{D}_{1}$, w.p. of failure $\delta \leq 1 / 3$. In this section, we prove that the communication cost of the protocol $\pi$ needs to be at least $\exp \left(k^{\Omega(\varepsilon)}\right)$ bits. Note that by (the easy direction of) Yao's minimax principle [34], we only need to consider deterministic protocols on the distribution $\mathcal{D}_{1}$ to prove this result.

The intuition behind the proof is as follows: By Lemma 3.4, the social welfare in the given instance changes by a factor of at least $k^{1-\varepsilon}$ depending on the value of $\theta$. This implies that any $k^{1-\varepsilon}=m^{\frac{1-\varepsilon}{3}}$ approximation algorithm for the social welfare can also determine the value of $\theta$. Using this, we can argue that the message sent by the players needs to reveal $\Omega(1)$ bit of information about the parameter $\theta$. Roughly speaking, this means that each of the $n$ players is responsible for revealing $\Omega(1 / n)$ bit about $\theta$ in average.

Furthermore, recall that the input to player $i \in N$ can be seen as a tuple $\left(x_{i}, \phi_{i}\right)$ (by Observation 3.2) and that $\theta=x_{i}\left(j^{\star}\right)$. Additionally, by Observation 3.3, given input $\left(x_{i}, \phi_{i}\right)$ to player $i$, the index $j^{\star}$ is chosen uniformly at random from $[p]$ and, hence, player $i$ is oblivious to which index of $x_{i}$ corresponds to the parameter $\theta$. This essentially means that player $i$ needs to reveal $\Omega(p / n)$ bits about the vector $x_{i}$ to be able to reveal $\Omega(1 / n)$ bit about $x_{i}\left(j^{\star}\right)$ (even when $j^{\star}$ is known to the referee), hence forcing $i$ to communicate $\Omega(p / n)=\exp \left(k^{\Omega(\varepsilon)}\right)$ bits also. To make the latter intuition precise, we argue that while the message sent by one player can, in principle, be used to infer information about the input of another player (as the input of the players are correlated), this extra information is limited to an "easy part" containing only $\left(\sigma, j^{\star}\right)$ that can even be assumed to be known to referee (but not players) beforehand. This allows us to "break" the information revealed to the referee to smaller pieces sent by each player, hence arguing that each player is indeed directly responsible for communicating the information about her input. We now formalize this intuition. We first need the following notation:

Notation. We use $\Pi=\left(\Pi_{1}, \ldots, \Pi_{n}\right)$ to denote the random variable for the transcript of the messages communicated in $\pi$. For any player $i \in N$, and any $j \in[p]$, we use the random variable $X_{i, j} \in\{0,1\}$ to denote the value of $x_{i}(j)$, i.e., $X_{i, j}=1$ iff the set $S_{j} \in \mathcal{S}$ is included in the private collection $\mathcal{F}_{i}$. We further define $X_{i}$ for $i \in N$ as the vector $X_{i}:=\left(X_{i, 1}, \ldots, X_{i, p}\right)$. We use $\Sigma$ to denote the random variable for the permutation $\sigma, J$ for the index $j^{\star}$, and $\Theta$ for the parameter $\theta$. For each player $i \in N, \Phi_{i}$ denotes the random variable for the labeling function $\phi_{i}$.

Recall that $(\Sigma, J)$ is the "easy part" of the input: the part that we assume the referee (but not each individual player) knows beforehand. Assuming this knowledge can only strengthen our lower 
bound. We start by arguing that the protocol $\pi$ needs to reveal $\Omega(1)$ bits of information about the value of parameter $\theta$ in the distribution.

CLAim 3.5. $\mathbb{I}(\Theta ; \Pi \mid \Sigma, J)=\Omega(1)$.

Proof. By Claim 3.4, the social welfare is $k^{1-\varepsilon}=m^{\frac{1-\varepsilon}{3}}$ times larger when $\theta=1$ than when $\theta=0$. Since $\pi$ outputs an $\left(m^{\frac{1-\varepsilon}{3}}\right)$-approximation to the social welfare, it can also be used to distinguish between the values of $\theta$ w.p. of error at most $\delta \leq 1 / 3$. This means that there is a function that, given the message $\Pi$ and variables $(\Sigma, J)$ (i.e., the easy part of the input), can determine the value of $\Theta$ w.p. of error at most $\delta$. This, together with Fano's inequality (Fact 2.4), implies that $\mathbb{H}(\Theta \mid$ $\Pi, \Sigma, J) \leq H_{2}(\delta)($ as $|\Theta|=2)$.

We now have,

$$
H_{2}(\delta) \geq \mathbb{H}(\Theta \mid \Pi, \Sigma, J)=\mathbb{H}(\Theta \mid \Sigma, J)-\mathbb{I}(\Theta ; \Pi \mid \Sigma, J)=1-\mathbb{I}(\Theta ; \Pi \mid \Sigma, J),
$$

where in the final equality, we used the fact that in $\mathcal{D}_{1}, \Theta$ is chosen uniformly at random from $\{0,1\}$ independent of $(\Sigma, J)$, and hence $\mathbb{H}(\Theta \mid \Sigma, J)=1$ (by Fact 2.3-(1)). To conclude, we have that $\mathbb{I}(\Theta ; \Pi \mid \Sigma, J) \geq 1-H_{2}(\delta)=\Omega(1)$ as $\delta$ is a constant bounded away from $1 / 2$.

We now show that the information revealed about $\Theta$ by the message $\Pi$ is at most the sum of information revealed by each message $\Pi_{i}$ for $i \in N$ individually. In other words, one does not gain an extra information by combining the messages of players (after conditioning on what is revealed by $(\Sigma, J)$ already).

Claim 3.6. $\mathbb{I}(\Theta ; \Pi \mid \Sigma, J) \leq \sum_{i \in N} \mathbb{I}\left(\Theta ; \Pi_{i} \mid \Sigma, J\right)$.

Proof. We have,

$$
\mathbb{I}(\Theta ; \Pi \mid \Sigma, J)=\sum_{i \in N} \mathbb{I}\left(\Theta ; \Pi_{i} \mid \Pi^{<i}, \Sigma, J\right) \leq \sum_{i \in N} \mathbb{I}\left(\Theta ; \Pi_{i} \mid \Sigma, J\right),
$$

where the equality is by chain rule (Fact 2.3-(4)), and the inequality follows from Proposition 2.6, as we show below that $\Pi_{i} \perp \Pi^{<i} \mid \Theta, \Sigma$, J, or equivalently $\mathbb{I}\left(\Pi_{i} ; \Pi^{<i} \mid \Theta, \Sigma, J\right)=0$ (by Fact 2.3-(2)).

As stated in Observation 3.2, the input of player $i \in N$ is uniquely determined by $\left(x_{i}, \phi_{i}\right)$ and, hence, $\Pi_{i}$ is a deterministic function of variables $X_{i}$ and $\Phi_{i}$. Moreover, $\Phi_{i}$ is also uniquely determined by $(\Sigma, J)$; hence, conditioned on $(\Sigma, J), \Pi_{i}$ is only a function of $X_{i}$. However, conditioned on $(\Theta, \Sigma, J), X_{i}$ and $X^{<i}$ are chosen independently of each other in the distribution $\mathcal{D}_{1}$ (as $X_{i, j^{\star}}=\theta$ and the rest of $X_{i}$ is chosen uniformly at random from $\left.\{0,1\}\right)$. This implies that $\mathbb{I}\left(X_{i} ; X^{<i}\right.$ $\Theta, \Sigma, J)=0$. As stated earlier, $\Pi_{i}$ is a function of $X_{i}$ and $\Pi^{<i}$ is a function of $X^{<i}$ alone (conditioned on $(\Theta, \Sigma, J))$, hence, by data processing inequality (Fact $2.3-(5)), \mathbb{I}\left(\Pi_{i} ; \Pi^{<i} \mid \Theta, \Sigma, J\right)=0$ as well, implying that inequality in the RHS above.

We now use a direct-sum style argument to prove that if a player $i \in N$ wants to communicate $c$ bits of information about $\theta$, she needs to communicate (essentially) $p \cdot c$ bits about her input.

Lemma 3.7. For any $i \in N, \mathbb{I}\left(\Theta ; \Pi_{i} \mid \Sigma, J\right) \leq\left|\Pi_{i}\right| / p$.

Proof. We first argue that $\mathbb{I}\left(\Theta ; \Pi_{i} \mid \Sigma, J\right) \leq \mathbb{I}\left(\Theta ; \Pi_{i} \mid \Phi_{i}, J\right)$. This is because $\Sigma$ and $J$ together uniquely determine the labeling function $\Phi_{i}$ and hence $\mathbb{I}\left(\Theta ; \Pi_{i} \mid \Sigma, J\right)=\mathbb{I}\left(\Theta ; \Pi_{i} \mid \Phi_{i}, \Sigma, J\right)$. Moreover, notice that $\Pi_{i} \perp \Sigma \mid \Phi_{i}$,J, because the input to player $i$ (and as a result $\Pi_{i}$ ) is identified by the labeling function $\Phi_{i}$ and vector $X_{i}$ (which is independent of $\Sigma$ ) by Observation 3.2. As such, dropping the conditioning on $\Sigma$ can only increase the mutual information by Proposition 2.5, implying that $\mathbb{I}\left(\Theta ; \Pi_{i} \mid \Sigma, J\right) \leq \mathbb{I}\left(\Theta ; \Pi_{i} \mid \Phi_{i}, J\right)$. We now have,

$$
\begin{array}{r}
\mathbb{I}\left(\Theta ; \Pi_{i} \mid \Phi_{i}, J\right)=\mathbb{E}_{j \in[p]}\left[\mathbb{I}\left(\Theta ; \Pi_{i} \mid \Phi_{i}, J=j\right)\right]=\mathbb{E}_{j \in[p]}\left[\mathbb{I}\left(X_{i, j} ; \Pi_{i} \mid \Phi_{i}, J=j\right)\right] \\
\left(\Theta=X_{i, j} \text { conditioned on } J=j\right)
\end{array}
$$




$$
=\frac{1}{p} \cdot \sum_{j \in[p]} \mathbb{I}\left(X_{i, j} ; \Pi_{i} \mid \Phi_{i}, J=j\right)=\frac{1}{p} \cdot \sum_{j \in[p]} \mathbb{I}\left(X_{i, j} ; \Pi_{i} \mid \Phi_{i}\right) .
$$

The last equality is because the joint distribution of $\left(X_{i, j}, \Pi_{i}, \Phi_{i}\right)$ is independent of the event $J=j$ by Observation 3.3 and, hence, we can drop the conditioning on this event. As such,

$$
\begin{aligned}
\mathbb{I}\left(\Theta ; \Pi_{i} \mid \Phi_{i}, J\right)=\frac{1}{p} \cdot \sum_{j \in[p]} \mathbb{I}\left(X_{i, j} ; \Pi_{i} \mid \Phi_{i}\right) \leq \frac{1}{p} \cdot \sum_{j \in[p]} \mathbb{I}\left(X_{i, j} ; \Pi_{i} \mid X_{i}^{<j}, \Phi_{i}\right) \\
\left.\quad \quad \text { (by Proposition } 2.5 \text { as } X_{i, j} \perp X_{i}^{<j} \mid \Phi_{i}\right) \\
=\frac{1}{p} \cdot \mathbb{I}\left(X_{i} ; \Pi_{i} \mid \Phi_{i}\right) \leq \frac{1}{p} \cdot \mathbb{H}\left(\Pi_{i} \mid \Phi_{i}\right) \leq \frac{1}{p} \cdot \mathbb{H}\left(\Pi_{i}\right) \leq \frac{1}{p} \cdot\left|\Pi_{i}\right|,
\end{aligned}
$$

where the equality in the second line is by chain rule (Fact 2.3-(4)), and inequalities are by Fact 2.3(1) and Fact 2.3-(3).

We can now conclude the following lemma:

Lemma 3.8. Communication cost of $\pi$ is $\Omega(p)$.

Proof. $\|\pi\|=\sum_{i \in N}\left|\Pi_{i}\right| \geq p \cdot \sum_{i \in N} \mathbb{I}\left(\Theta ; \Pi_{i} \mid \Sigma, J\right) \geq p \cdot \mathbb{I}(\Theta ; \Pi \mid \Sigma, J)=\Omega(p)$, where the last three equations are by, respectively, Lemma 3.7, Claim 3.6, and Claim 3.5.

Theorem 2 now follows from Lemma 3.8 by re-parameterizing $\varepsilon$ above by some $\Theta(\varepsilon)$ and noting that $p=\exp \left(\Theta\left(k^{\varepsilon}\right)\right)=\exp \left(m^{\Omega(\varepsilon)}\right)\left(\right.$ as $\left.m=2 k^{3}\right)$.

\section{MAIN RESULT: A LOWER BOUND FOR MULTI-ROUND PROTOCOLS}

In this section, we establish our main result. Formally,

THEOREM 3. For any integer $1 \leq r \leq o\left(\frac{\log m}{\log \log m}\right)$ and any sufficiently small constant $\varepsilon>0$, any $r$ round protocol (possibly randomized) for combinatorial auctions with subadditive (even XOS) bidders that can approximate the value of social welfare to a factor of $\left(\frac{1}{r} \cdot m^{\frac{1-\varepsilon}{2 r+1}}\right)$ w.p. at least $2 / 3$ requires $\exp \left(m^{\Omega\left(\frac{\varepsilon}{r}\right)}\right)$ bits of communication in the worst case.

We start by introducing the recursive family of hard input distributions that we use in proving Theorem 3 in Section 4.1 and then establish a lower bound for this distribution in Section 4.2.

\subsection{A Hard Input Distribution for $\boldsymbol{r}$-Round Protocols}

Our hard distribution $\mathcal{D}_{r}$ for $r$-round protocols is defined recursively such that its base case ( $r=1$ case) is the distribution $\mathcal{D}_{1}$ introduced in Section 3.1. We first give an informal description of $\mathcal{D}_{r}$.

Let $k$ be an integer and consider a set $N$ of $n_{r}=k^{2 r}$ players and a set $M$ of $m_{r}=(r+1) \cdot k^{2 r+1}$ items. The players are partitioned (arbitrarily) between $k^{2}$ groups $N_{1}, \ldots, N_{k^{2}}$ each of size $n_{r-1}$. Fix a group $N_{g}$ and for any player $i \in N_{g}$, we create an exponentially large (in $k$ ) collection $C_{i}$ of item-sets of size $m_{r-1}$ (over the universe $M$ ), such that for any two sets $S, T \in C_{i},|S \cap T| \leq k^{2 r-2+\varepsilon}$ (for any constant $\varepsilon>0$ ).

The local view of player $i \in N_{g}$ is as follows: Over each set $S_{j} \in C_{i}$, we create an $(r-1)$-round instance of the problem-namely, instance $I_{i, j}$, sampled from the distribution $\mathcal{D}_{r-1}$ with the set of players $N_{g}$ and the set of items $S_{j}$-and then let the input of player $i$ be the collective input of the $i$ th player in all these instances. In other words, player $i$ finds herself "playing" in exponentially many " $(r-1)$-round instances" of $\mathcal{D}_{r-1}$.

On the group level, the inputs to players inside a group $N_{g}$ are highly correlated: For each player $i \in N_{g}$, one of the instances (namely, $I_{i, j^{\star}}$ ) is a "special instance" in the sense that all players in the group $N_{g}$ has a "consistent" view of this instance, i.e., the collective view of players $1, \ldots, n_{r-1}$ in 
$N_{g}$ on the instances $I_{1, j^{\star}}, \ldots, I_{n_{r-1}, j^{\star}}$ forms a valid instance sampled from $\mathcal{D}_{r-1}$. However, for any other index $j \neq j^{\star}$, the collective view of players in $N_{g}$ in the instances $I_{1, j^{\star}}, \ldots, I_{n_{r-1}, j^{\star}}$ forms a "pseudo instance" that is not sampled from $\mathcal{D}_{r-1}$; these pseudo instances are created by sampling the input of each player independently according to $\mathcal{D}_{r-1}$. Note, however, that while the pseudo instances and the special instance of a player are fundamentally different, each player is oblivious to this difference, i.e., cannot determine (locally) which instance is the special instance.

Finally, the input to players across the groups, i.e., the global input, is further correlated: The set of items in the special instances of players in a group $N_{g}$ is a "unique" set of items (across all groups), while all other instances, across all groups, are constructed over a set of $k^{2 r}$ "shared" items. This correlation makes the special instance of a player $i$, in some sense, the only important instance: To obtain a large allocation, the players need to ultimately solve the problem for these special instances.

We now formally define distribution $\mathcal{D}_{r}$. In the following, for simplicity of exposition, we assume that the distribution $\mathcal{D}_{r}$, in addition to the valuation function of players, also outputs the private collections (defined similarly as in $\mathcal{D}_{1}$ ) of players that are used to define these functions. ${ }^{4}$

Distribution $\mathcal{D}_{r}(N, M)$. A hard input distribution for $r$-round protocols (for $r \geq 2$ ).

Input: Collections $N$ of $n_{r}=k^{2 r}$ players and $M$ of $m_{r}=(r+1) \cdot k^{2 r+1}$ items.

Output: A set of $n_{r}$ valuation functions $\left(v_{1}, \ldots, v_{n_{r}}\right)$ for the players in $N$ and $n_{r}$ private collections $\left(\mathcal{F}_{1}, \ldots, \mathcal{F}_{n_{r}}\right)$ used to define the valuation functions.

(1) Let $\mathcal{S}_{r}=\left\{S_{1}, \ldots, S_{p}\right\}$ be a $\left(p_{r}, q_{r}, t_{r}, \ell_{r}\right)$-intersecting family with parameters $p_{r}=p=$ $\exp \left(\Theta\left(k^{\varepsilon}\right)\right), q_{r}=k^{2 r}+r \cdot k^{2 r-1}, t_{r}=r \cdot k^{2 r-1}$, and $\ell_{r}=k^{2 r-2+\varepsilon}$ (guaranteed to exist by Lemma 2.2 as $k=m^{\Omega(1 / r)}=\omega\left(r^{2 / \varepsilon}\right)$ by the assumption that $r=o\left(\frac{\log m}{\log \log m}\right)$; we emphasize that even though Lemma 2.2 in fact guarantees such a collection for a much larger choice of $p$, we only need the value $p=\exp \left(\Theta\left(k^{\varepsilon}\right)\right)$ in our proof).

(2) Arbitrarily group the players into $k^{2}$ groups $\mathcal{N}=\left(N_{1}, \ldots, N_{k^{2}}\right)$, whereby each group contains exactly $n_{r-1}=k^{2 r-2}$ players.

(3) Pick an index $j^{\star} \in[p]$ uniformly at random and sample an instance $I_{r}^{\star} \sim$ $\mathcal{D}_{r-1}\left(\left[n_{r-1}\right], S_{j^{\star}}\right)$.

(4) For each group $N_{g} \in \mathcal{N}$ independently,

(a) Define $I_{N_{g}}^{\star}$ as $I_{r}^{\star}$ by mapping the players in $\left[n_{r-1}\right]$ to $N_{g}$.

(b) For each player $i \in N_{g}$ independently, create $p$ instances $I^{(i)}:=\left(I_{i, 1}, \ldots, I_{i, p}\right)$ whereby for all $j \neq j^{\star}, I_{i, j} \sim \mathcal{D}_{r-1}\left(N_{g}, S_{j}\right)$, and $I_{i, j^{\star}}=I_{N_{g}}^{\star}$.

(c) For a player $i \in N_{g}$ and index $j \in[p]$, let $\mathcal{F}_{i, j}$ be the set of private collection of that player in instance $I_{i, j}$ and let $\mathcal{F}_{i}=\bigcup_{j \in[p]} \mathcal{F}_{i, j}$.

(5) Pick a random permutation $\sigma$ of $M$. For each $g \in\left[k^{2}\right]$ and group $N_{g}$, map the $k^{2 r}$ items in $\left[q_{r}\right] \backslash S_{j^{\star}}$ to $\sigma(1), \ldots, \sigma\left(k^{2 r}\right)$, and the $t_{r}$ items in $S_{j^{\star}}$ to $\sigma\left(k^{2 r}+(g-1) \cdot t_{r}+1\right) \ldots \sigma(g$. $t_{r}$ ) (and for each player $i \in N_{g}$, update the item set of $\mathcal{F}_{i}$ and underlying instances $I_{i, 1}, \ldots, I_{i, p}$ accordingly).

(6) For any player $i \in N$, define the valuation function of player $i$ as $v_{i}(S)=\max _{T \in \mathcal{F}_{i}}|S \cap T|$ (note that these valuation functions are XOS valuation; see Observation 3.1).

We make several observations about the distribution $\mathcal{D}_{r}$. Recall that $\mathcal{F}_{i}$ denotes the private collection of player $i \in N$ that is used to define the valuation function $v_{i}$. By construction, the size

${ }^{4}$ Strictly speaking, this is a redundant information, as the valuation functions can uniquely determine the private collections; however, we include this redundant output for the ease of presentation. 
of the sets inside each private collection is equal across any two distributions $\mathcal{D}_{r}$ and $\mathcal{D}_{r^{\prime}}$ and hence is equal to $k$ (by definition of distribution $\mathcal{D}_{1}$ ). A simple property of these sets is that,

Observation 4.1. For any player $i \in N$, and any set $T \in \mathcal{F}_{i}$, the set $T$ is chosen uniformly at random from all $k$-subsets of $M$.

Fix any group $N_{g} \in \mathcal{N}$ and any player $i \in N_{g}$. The input to player $i$ can be seen as the "view" of $i$ in the $p$ instances $I^{(i)}:=\left(I_{i, 1}, \ldots, I_{i, p}\right)$, i.e., the input of the $i$ th player (in $\left.N_{g}\right)$ in $I_{i, j}$ (for all $j \in[p]$ ) and not the whole instance. However, in the following, we slightly abuse the notation and use $I_{i, j}$ to also denote the view of player $i$ in the instance $I_{i, j}$. Moreover, we point out that $I_{i, j}$ is defined over the set of items $S_{j}$; hence, the complete input to player $i$ is the pair $\left(I^{(i)}, \phi_{i}\right)$ where $\phi_{i}$ is the labeling function to map the items in $\bigcup_{j \in[p]} S_{j}$ to $M$ (see also Observation 3.2).

For any player $i \in N$, we refer to the instance $I_{i, j^{\star}}$ of player $i$ as the special instance of player $i$, and to all other instances $I_{i, j}$ for $j \neq j^{\star}$ as fooling instances. We further define $I_{r}^{\star}(i)$ as the input of player $i$ in the special instance $I_{i, j^{\star}}=I_{r}^{\star}$ and define $I_{r}^{\star}(-i)$ as the input of all other players in $I_{r}^{\star}$.

ObSERvation 4.2. For any group $N_{g} \in \mathcal{N}$, the joint input of all players $i \in N_{g}$ in their special instances $I_{i, j^{\star}}$ form the instance $I_{N_{g}}^{\star}$ that is sampled from the distribution $\mathcal{D}_{r-1}$.

However, the fooling instances of players $i \in N_{g}$ are sampled independently and, hence, the joint distribution of the players on their instances $I_{i, j}$ is not sampled from $\mathcal{D}_{r-1}$. Nevertheless, this difference is not evident to the player $i$.

ObSeRvation 4.3. For any player $i \in N$, conditioned on the input $\left(I^{(i)}, \phi_{i}\right)$ given to the player $i$, the index $j^{\star}$ is chosen uniformly at random from $[p]$.

Additionally,

OBSERvation 4.4. The distribution of collection of instances $\mathcal{I}:=\left(I^{(1)}, \ldots, I^{\left(n_{r}\right)}\right) \sim \mathcal{D}_{r} \mid I_{r}^{\star}, \sigma, j^{\star}$ is a product distribution, as instances in Line (4b) are sampled independently (except for instances $I_{i, j^{\star}}=I_{r}^{\star}$, which are already conditioned on above).

Another important property of the special instances in distribution $\mathcal{D}_{r}$ is that,

OBservation 4.5. The special instances $I_{N_{1}}^{\star}, \ldots, I_{N_{k^{2}}}^{\star}$ are supported on disjoint set of items (according to the mapping $\sigma$ ).

Notice that we can trace the special instances into a unique path $I_{r}^{\star} \rightarrow I_{r-1}^{\star} \rightarrow \cdots \rightarrow I_{2}^{\star}$, whereby $I_{2}^{\star}$ is sampled from the distribution $\mathcal{D}_{1}$. We use $\theta^{\star}$ to denote the parameter $\theta$ (in $\mathcal{D}_{1}$ ) in the instance $I_{2}^{\star}$ in this path. The following lemma proves a key relation between $\theta^{\star}$ and social welfare of the sampled instance:

LEMMA 4.6. For any instance $I \sim \mathcal{D}_{r}$ :

$$
\begin{aligned}
& \operatorname{Pr}\left(\operatorname{sw}(I) \geq k^{2 r+1} \mid \theta^{\star}=1\right)=1, \\
& \operatorname{Pr}\left(\operatorname{sw}(I) \leq 2 r \cdot k^{2 r+2 \varepsilon} \mid \theta^{\star}=0\right)=1-r \cdot \exp \left(-\Omega\left(k^{\varepsilon}\right)\right) .
\end{aligned}
$$

Proof. We start by the simpler case of Equation (1); the proof is by induction. The base case, when $r=1$, is true by Lemma 3.4. Suppose this holds for all integers smaller than $r$. Now, consider an instance $I \sim\left(\mathcal{D}_{r} \mid \theta^{\star}=1\right)$ and the $k^{2}$ special instances $I_{N_{1}}, \ldots, I_{N_{k^{2}}}$ sampled from $\left(\mathcal{D}_{r-1} \mid \theta^{\star}=\right.$ 1) in $I$. By induction, there is an allocation $A_{g}$ for each $g \in\left[k^{2}\right]$ that results in a welfare of at least $k^{2 r-1}$ in each $I_{N_{g}}^{\star}$. By Observation 4.5, the set of items among special instances are disjoint, and hence the allocation $A:=\left(A_{1}, \ldots, A_{k^{2}}\right)$ that assigns the bundles in $A_{g}$ to players in $N_{g}$ for $g \in\left[k^{2}\right]$ is a valid allocation that results in a welfare of $k^{2} \cdot k^{2 r-1}=k^{2 r+1}$, proving the induction step. 
We now prove Equation (2) by induction. The base case of $r=1$ is true by Lemma 3.4. Assume that the bounds hold for all integers smaller than $r$ and consider an instance $I \sim\left(\mathcal{D}_{r} \mid \theta^{\star}=0\right)$ and let $I_{N_{1}}^{\star}, \ldots, I_{N_{k^{2}}}^{\star}$ be the special instances of $I$, "copied" from the instance $I_{r}^{\star} \sim\left(\mathcal{D}_{r-1} \mid \theta^{\star}=0\right.$ ) (as in Line (4a) of $\mathcal{D}_{r}$ ). Let $U$ be the set of items assigned to these instances (by mapping $\sigma$ ) and $\bar{U}$ be the set of remaining items assigned by $\sigma$, i.e., the items that have no value in the special instances; we have $|U|=k^{2} \cdot t_{r}=r \cdot k^{2 r+1}$ and $|\bar{U}|=k^{2 r}$. (Notice that $\sigma$ does not assign all the items in $M$; in particular, $k^{2 r+1}-k^{2 r}$ items are not assigned to any instance, i.e., have no value for any player; these extra items are only added to simplify the math.) We have,

Claim 4.7. W.p. $1-\exp \left(-\Omega\left(k^{\varepsilon}\right)\right)$ for any player $i \in N$ and any set $T \in F_{i}$ such that $T$ does not belong to a private collection of a special instance (i.e., $T$ is not sampled from $I_{i, j^{\star}}$ ), $|T \cap U| \leq k^{2 \varepsilon}$.

Proof. Fix a group $N_{g} \in \mathcal{N}$ and fix a player $i \in N_{g}$ and let $I_{i, j}$ be an instance of $\mathcal{D}_{r-1}$ for some $j \neq j^{\star}$, i.e., not a special instance. Recall that the set of items in $I_{i, j}$ and $I_{i, j^{\star}}$ are two distinct sets $S_{j}$ and $S_{j^{\star}}$ from $\mathcal{S}_{r}$ on the universe $\left[q_{r}\right]$ (and hence $\left|S_{j} \cap S_{j^{\star}}\right| \leq \ell_{r}=k^{2 r-2+\varepsilon}$ by definition of intersecting families) and, since $\left[q_{r}\right]$ is entirely mapped by $\sigma$ for player $i \in N_{g}$, the intersection between item set of $I_{i, j}$ and $I_{i, j^{\star}}$ is at most $k^{2 r-2+\varepsilon}$; this in particular means that at most $k^{2 r-2+\varepsilon}$ items in $I_{i, j}$ belong to $U\left(I_{i, j}\right.$ does not share any item with any instance $I_{i^{\prime}, j^{\star}}$ for any $\left.i^{\prime} \notin N_{g}\right)$.

Now consider the choice of a set $T$ (in the private collection) for the player $i$ in the instance $I_{i, j}$. For each item $a$ that belongs to both item-set of $I_{i, j}$ and $U$, define an indicator random variable $X_{a} \in\{0,1\}$, which is one iff $a$ is chosen in $T$. Then, $X:=\sum_{a} X_{a}$ denotes $|T \cap U|$. By Observation 4.1, $T$ is a $k$-subset chosen uniformly at random from a universe of size $t_{r}=r \cdot k^{2 r-1}$, and hence, $\mathbb{E}[X] \leq k^{2 r-2+\varepsilon} \cdot 1 /\left(r \cdot k^{2 r-2}\right) \leq k^{\varepsilon} / r$. By Chernoff bound for negatively correlated random variables (Proposition 2.1), $\operatorname{Pr}\left(|S \cap U| \geq k^{2 \varepsilon}\right) \leq \exp \left(-\Omega\left(k^{2 \varepsilon}\right)\right)$.

We can now apply a union bound for all possible choices for the set $T$ (among all players and instances), and the probability that even one set $T$ violates this constraint is (note that there are $n_{r} \cdot p^{r}$ different choices for $T$ )

$$
n_{r} \cdot p^{r} \cdot \exp \left(-\Omega\left(k^{2 \varepsilon}\right)\right)=\exp (\Theta(r \cdot \log k)) \cdot \exp \left(\Theta\left(r \cdot k^{\varepsilon}\right)\right) \cdot \exp \left(-\Omega\left(k^{2 \varepsilon}\right)\right)=\exp \left(-\Omega\left(k^{\varepsilon}\right)\right),
$$

since $r=o\left(k^{\varepsilon}\right)$ (by the assumption that $r=o\left(\frac{\log m}{\log \log m}\right)$ ).

In the following, we condition on the event in Claim 4.7 (event $\mathcal{E}_{1}$ ) and the event that $\operatorname{sw}\left(I^{\star}\right) \leq$ $2(r-1) \cdot k^{2 r-2+2 \varepsilon}\left(\right.$ event $\left.\mathcal{E}_{2}\right)$. Note that by Claim 4.7 and induction hypothesis, these two events happen (simultaneously) w.p. $1-r \cdot \exp \left(-\Omega\left(k^{\varepsilon}\right)\right)$.

Now fix any allocation $\mathcal{A}=\left(A_{1}, \ldots, A_{n}\right)$. As size of $\bar{U}$ is at most $k^{2 r}$, the items in $\bar{U}$ can only contribute $k^{2 r}$ to the welfare in $\mathcal{A}$. Next, let $\mathcal{A}^{*}$ be the subset of $\mathcal{A}$ such that the maximizing clause in each $A_{i} \in \mathcal{A}^{*}$ (i.e., the set $T \in \mathcal{F}_{i}$ ) belongs to some special instance, and $\mathcal{A}^{\prime}$ be the remaining part of allocation $\mathcal{A}$. We know, by $\mathcal{E}_{2}$, that the contribution of $\mathcal{A}^{*}$ to the welfare is at most $k^{2} \cdot 2(r-$ $1) \cdot k^{2 r-2+2 \varepsilon}=2(r-1) \cdot k^{2 r+2 \varepsilon}$ (counting the $k^{2}$ special instances). Moreover, by $\mathcal{E}_{1}$ (in Claim 4.7), the contribution of $\mathcal{A}^{\prime}$ is at most $k^{2 r} \cdot k^{2 \varepsilon}=k^{2 r+2 \varepsilon}$. To conclude, we obtain that the social welfare when $\theta^{\star}=0$ is at most $k^{2 r}+2(r-1) \cdot k^{2 r+2 \varepsilon}+k^{2 r+2 \varepsilon} \leq 2 r \cdot k^{2 r+2 \varepsilon}$ with the desired probability, proving the lemma.

\subsection{The Lower Bound for Distribution $\mathcal{D}_{r}$}

Let $\pi$ be a $r$-round protocol that can output a $\left(\frac{1}{4 r} \cdot m_{r}^{\frac{1-2 \varepsilon}{2 r+1}}\right)$-approximation to the social welfare of any instance $I \sim \mathcal{D}_{r}$, w.p. of failure $\delta<1 / 4$. In this section, we prove that the communication cost of the protocol $\pi$ needs to be at least $\exp \left(\Omega\left(k^{\varepsilon}\right)\right)$ bits. By (the easy direction of) Yao's minimax principle [34], it suffices to prove this lower bound for deterministic algorithms. 
We start by providing a detailed overview of the proof. First, by Lemma 4.6, we can argue that the protocol $\pi$ is also a $(\delta+o(1))$-error protocol for estimating the parameter $\theta^{\star}$ and, hence, we prove the lower bound for $\theta^{\star}$-estimation problem instead. Recall that in any instance $I_{r} \sim \mathcal{D}_{r}$, the value of $\theta^{\star}$ is equal to the value of $\theta^{\star}$ in the underlying special instance $I_{r}^{\star}$ in $I_{r}$, and that $I_{r}^{\star}$ is sampled from the distribution $\mathcal{D}_{r-1}$. Hence, to "solve" the instance $I_{r} \sim \mathcal{D}_{r}$, the players need to be able to solve the instance $I_{r}^{\star} \sim \mathcal{D}_{r-1}$ as well. This suggests an inductive approach to prove the lower bound for the distribution $\mathcal{D}_{r}$.

Consider the first message $\Pi_{1}=\left(\Pi_{1,1}, \ldots, \Pi_{1, n_{r}}\right)$ of $\pi$. Recall that the input to any player $i \in N$ consists of $p$ different instances (of $\mathcal{D}_{r-1}$ ), one of which is the instance $I_{r}^{\star}$. By Observation 4.3, each player $i$ is oblivious to the identity of $I_{r}^{\star}$ and hence, intuitively, the message $\Pi_{1, i}$ cannot reveal more than $\approx\left|\Pi_{1, i}\right| / p$ bits of information about the instance $I_{r}^{\star}$. Considering the simultaneity of the protocol $\pi$, we can use a similar argument as in the previous section and prove that if $\left|\Pi_{1}\right|=o(p)$, then at most $o(1)$ bits of information is revealed about $I_{r}^{\star}$.

Now consider the second round of the protocol $\pi$. The task of players in each group $N_{g} \in \mathcal{N}$ is now to solve the instance $I_{r}^{\star}$ (on a separate set of players and items). As argued above, the first message of players can only reveal $o(1)$ bits of information about $I_{r}^{\star}$ and, hence, distribution of $I_{r}^{\star}$ is still "very close" to its original distribution $\mathcal{D}_{r-1}$, even conditioned on the first message of players. But $\mathcal{D}_{r-1}$ is assumed inductively to be a hard input distribution for $(r-1)$-round protocols and as $\pi$ needs to solve $I_{r}^{\star}$ in $(r-1)$ rounds now, we may argue that it needs an exponential communication.

To make this intuition precise, we employ a round-elimination argument: Given any hard instance $I_{r-1} \sim \mathcal{D}_{r-1}$, we "embed" $I_{r-1}$ in an $r$-round instance $I_{r}$ sampled from $\mathcal{D}_{r}$ conditioned on the first message $\Pi_{1}$ of $\pi$ with no communication between the players and then use $\pi$ from the second round onwards to solve $I_{r-1}$. However, notice that as the number of players (and items) varies between $I_{r}$ and $I_{r-1}$, we cannot directly apply $\pi$ on $I_{r-1}$. Instead, the players first sample a message $\Pi_{1}$ (of $\pi$ ) according to the distribution $\mathcal{D}_{r}$ using public randomness. Next, each player $i \in\left[n_{r-1}\right]$ in the instance $I_{r-1}$ mimics the role of $k^{2}$ different players (one "copy" in each group in $\mathcal{N}$ in $I_{r}$ ) by letting the input of each copy in the special instance (of $I_{r}$ ) be her input in $I_{r-1}$ and then "completes" the rest of her input (i.e., her fooling instances in $I_{r}$ ) independently of other players to obtain an instance $I_{r} \sim \mathcal{D}_{r} \mid I_{r}^{\star}=I_{r-1}, \Pi_{1}$. Note that a priori it is not clear why such an embedding is possible, since the first message $\Pi_{1}$ correlates the input of players in fooling instances, making independent sampling of these instances impossible. However, we show that by further conditioning on some "easy part" of the input in the first round, i.e., $\sigma$ and $j^{\star}$ (by sampling these parts publicly also), the players can indeed implement this embedding without any communication and hence obtain a valid $(r-1)$-round protocol for $I_{r-1}$. We are now ready to present the formal proof. To continue, we need the following notation:

Notation. For any $j \in[r]$, we use $\Pi_{j}=\left(\Pi_{j, 1}, \ldots, \Pi_{j, n_{r}}\right)$ to denote the random variable for the transcript of the messages communicated in the round $j$ of $\pi$. For any player $i \in N$ and any $j \in[p]$, we override the notation and use $I_{i, j}$ to also denote the random variable for the instance $I_{i, j}$ sampled in $\mathcal{D}_{r}$ (similarly for $I_{r}^{\star}$ and $\left.I^{(i)}\right)$. We further use $\Sigma$ to denote the random variable for the permutation $\sigma$ and $J$ for the index $j^{\star}$. We start by the following simple claim:

Claim 4.8. Protocol $\pi$ can also determine the value of $\theta^{\star}$ w.p. $1-\delta-o(1)$.

Proof. By Lemma 4.6, the ratio of $\operatorname{sw}(I)$ depending on the parameter $\theta^{\star}$ is (w.p. $1-o(1)$ ):

$$
\frac{k^{2 r+1}}{2 r \cdot k^{2 r+2 \varepsilon}}=\frac{k^{1-2 \varepsilon}}{2 r}=\frac{m_{r}^{\frac{1-2 \varepsilon}{2+1}}}{2 r \cdot(r+1)^{\frac{1-2 \varepsilon}{2 r+1}}}>\frac{1}{4 r} \cdot m_{r}^{\frac{1-2 \varepsilon}{2 r+1}} \text {. }
$$


Hence, the $\delta$-error $\left(\frac{1}{4 r} \cdot m_{r}^{\frac{1-2 \varepsilon}{2+1}}\right)$-approximation protocol $\pi$ correctly determines the value of $\theta^{\star}$ w.p. $1-\delta-o(1)$.

We show that as long as the first message sent by the players is not too large, this message cannot reveal much information about the special instance $I_{r}^{\star}$ embedded in the distribution $\mathcal{D}_{r}$. This argument is similar to the one in Section 3.2.

Lemma 4.9. If $\left|\Pi_{1}\right|=o\left(p / r^{4}\right)$, then $\mathbb{I}\left(I_{r}^{\star} ; \Pi_{1} \mid \Sigma, J\right)=o\left(1 / r^{4}\right)$.

We break the proof of Lemma 4.9 into two separate steps. First, we argue that the information revealed by the first message about $I_{r}^{\star}$ is at most the total summation of the information revealed by each individual player about $I_{r}^{\star}$, after conditioning on the "easy part" of the input in the first round, i.e., $\sigma$ and $j^{\star}$. The proof of this claim is essentially identical to that of Claim 3.6 and is provided for completeness.

ClaIm 4.10. $\mathbb{I}\left(I_{r}^{\star} ; \Pi_{1} \mid \Sigma, J\right) \leq \sum_{i \in N} \mathbb{I}\left(I_{r}^{\star}(i) ; \Pi_{1, i} \mid \Sigma, J\right)$.

Proof. We have,

$$
\mathbb{I}\left(I_{r}^{\star} ; \Pi_{1} \mid \Sigma, J\right)=\sum_{i \in N} \mathbb{I}\left(I_{r}^{\star} ; \Pi_{1, i} \mid \Pi_{1}^{<i}, \Sigma, J\right) \leq \sum_{i \in N} \mathbb{I}\left(I_{r}^{\star} ; \Pi_{1, i} \mid \Sigma, J\right),
$$

where the equality is by chain rule (Fact 2.3-(4)), and the inequality follows from Proposition 2.6, as we prove below that $\Pi_{1, i} \perp \Pi_{1}^{<i} \mid I_{r}^{\star}, \Sigma, J$ or equivalently $\mathbb{I}\left(\Pi_{1, i} ; \Pi_{1}^{<i} \mid I_{r}^{\star}, \Sigma, J\right)=0$ (by Fact 2.3(2)).

Define $\mathcal{I}:=\left(I^{(1)}, \ldots, I^{\left(n_{r}\right)}\right)$. As stated in Observation 4.4, we have $\mathcal{I}_{i} \perp \mathcal{I}^{<i} \mid I_{r}^{\star}, \Sigma, J$ and hence $\mathbb{I}\left(\mathcal{I}_{i} ; \mathcal{I}^{<i} \mid I_{r}^{\star}, \Sigma, J\right)=0$ (by Fact 2.3-(2)). Moreover, notice that for any player $i \in N, \Pi_{1, i}$ is a deterministic function of $I^{(i)}, \Sigma, J$ and hence, conditioned on $I_{r}^{\star}, \Sigma, J$, message $\Pi_{1, i}$ is only a function of $\mathcal{I}_{i}=I^{(i)}$. Consequently, by data processing inequality (Fact $\left.2.3-(5)\right)$, we also have $\mathbb{I}\left(\Pi_{1, i} ; \Pi_{1}^{<i}\right.$ $\left.I_{r}^{\star}, \Sigma, J\right)=0$.

Finally, we argue that $\mathbb{I}\left(I_{r}^{\star} ; \Pi_{1, i} \mid \Sigma, J\right)=\mathbb{I}\left(I_{r}^{\star}(i) ; \Pi_{1, i} \mid \Sigma, J\right)$ for all $i \in N$, which concludes the proof. This is because $I_{r}^{\star}=\left(I_{r}^{\star}(i), I_{r}^{\star}(-i)\right)$ and hence by chain rule of mutual information (Fact 2.3(4)), we have,

$$
\mathbb{I}\left(I_{r}^{\star} ; \Pi_{1, i} \mid \Sigma, J\right)=\mathbb{I}\left(I_{r}^{\star}(i) ; \Pi_{1, i} \mid \Sigma, J\right)+\mathbb{I}\left(I_{r}^{\star}(-i) ; \Pi_{1, i} \mid I_{r}^{\star}(i), \Sigma, J\right),
$$

as $\mathbb{I}\left(I_{r}^{\star}(-i) ; \Pi_{1, i} \mid I_{r}^{\star}(i), \Sigma, J\right)=0$, since $\Pi_{1, i}$ is independent of $I_{r}^{\star}(-i)$ after conditioning on $I_{r}^{\star}(i)$ and $\Sigma, J$ (as the input to player $i$ is independent of $I_{r}^{\star}(-i)$ after conditioning on $I_{r}^{\star}(i), \Sigma, J$ ) and, hence, we can apply Fact 2.3-(2).

In the next step, we use a direct-sum style argument to show that,

Lemma 4.11. For any $i \in N, \mathbb{I}\left(I_{r}^{\star}(i) ; \Pi_{1, i} \mid \Sigma, J\right) \leq\left|\Pi_{1, i}\right| / p$.

Proof. We first argue that $\mathbb{I}\left(I_{r}^{\star}(i) ; \Pi_{1, i} \mid \Sigma, J\right) \leq \mathbb{I}\left(I_{r}^{\star}(i) ; \Pi_{1, i} \mid \Phi_{i}, J\right)$. This is because $\Sigma$ and $J$ uniquely determine the labeling function $\Phi_{i}$ and hence $\mathbb{I}\left(I_{r}^{\star}(i) ; \Pi_{1, i} \mid \Sigma, J\right)=\mathbb{I}\left(I_{r}^{\star}(i) ; \Pi_{1, i} \mid\right.$ $\left.\Phi_{i}, \Sigma, J\right)$. Moreover, notice that $\Pi_{1, i} \perp \Sigma \mid \Phi_{i}, J$, because the input to player $i$ (and as a result $\Pi_{1, i}$ ) is identified by the labeling function $\Phi_{i}$ and instances $I^{(i)}$ (which are independent of $\Sigma$ ). As such, dropping the conditioning on $\Sigma$ can only increase the mutual information by Proposition 2.5, implying that $\mathbb{I}\left(I_{r}^{\star}(i) ; \Pi_{1, i} \mid \Sigma, J\right) \leq \mathbb{I}\left(I_{r}^{\star}(i) ; \Pi_{1, i} \mid \Phi_{i}, J\right)$. We now have, 


$$
\begin{aligned}
& \mathbb{I}\left(I_{r}^{\star}(i) ; \Pi_{1, i} \mid \Phi_{i}, J\right)=\mathbb{E}_{j \in[p]}\left[\mathbb{I}\left(I_{r}^{\star}(i) ; \Pi_{1, i} \mid \Phi_{i}, J=j\right)\right]= \mathbb{E}_{j \in[p]}\left[\mathbb{I}\left(I_{i, j} ; \Pi_{1, i} \mid \Phi_{i}, J=j\right)\right] \\
&\left(I_{r}^{\star}(i)=I_{i, j} \text { conditioned on } J=j\right) \\
&=\frac{1}{p} \cdot \sum_{j=1}^{p} \mathbb{I}\left(I_{i, j} ; \Pi_{1, i} \mid \Phi_{i}, J=j\right)=\frac{1}{p} \cdot \sum_{j=1}^{p} \mathbb{I}\left(I_{i, j} ; \Pi_{1, i} \mid \Phi_{i}\right) .
\end{aligned}
$$

The last equality is because the joint distribution of $\left(I_{i, j}, \Pi_{1, i}, \Phi_{i}\right)$ is independent of the event $J=j$ by Observation 4.3 and, hence, we can drop the conditioning on this event. As such,

$$
\begin{aligned}
\mathbb{I}\left(I_{r}^{\star}(i) ; \Pi_{1, i} \mid \Phi_{i}, J\right) & =\frac{1}{p} \cdot \sum_{j=1}^{p} \mathbb{I}\left(I_{i, j} ; \Pi_{1, i} \mid \Phi_{i}\right) \\
& \leq \frac{1}{p} \cdot \sum_{j=1}^{p} \mathbb{I}\left(I_{i, j} ; \Pi_{1, i} \mid I^{(i)<j}, \Phi_{i}\right) \quad\left(\text { by Proposition } 2.5 \text { as } I_{i, j} \perp I^{(i)<j} \mid \Phi_{i}\right) \\
& =\frac{1}{p} \cdot \mathbb{I}\left(I^{(i)} ; \Pi_{1, i} \mid \Phi_{i}\right) \leq \mathbb{H}\left(\Pi_{1, i}\right) / p \leq\left|\Pi_{1, i}\right| / p,
\end{aligned}
$$

where the equality is by chain rule (Fact 2.3-(4)) and final inequality is by Fact 2.3-(1).

We now have,

Proof of Lemma 4.9 By Claim 4.10 and Lemma 4.11,

$$
\mathbb{I}\left(I_{r}^{\star} ; \Pi_{1} \mid \Sigma, J\right) \leq \sum_{i \in N} \mathbb{I}\left(I_{r}^{\star}(i) ; \Pi_{1, i} \mid \Sigma, J\right) \leq \sum_{i \in N}\left|\Pi_{1, i}\right| / p=\left|\Pi_{1}\right| / p=o\left(1 / r^{4}\right)
$$

by the lemma assumption that $\left|\Pi_{1}\right|=o\left(p / r^{4}\right)$.

Recall that $I_{r}^{\star}$ is the special instance in distribution $\mathcal{D}_{r}$, which was sampled from distribution $\mathcal{D}_{r-1}$. We define $\psi_{r}$ as the distribution of $I_{r}^{\star}$ conditioned on $\left(\Pi_{1}, \Sigma, J\right)$, i.e., after seeing the first message of $\pi$ and the easy part of the input $(\Sigma, J)$. As a corollary of Lemma 4.9, we have that this further conditioning does not change the distribution of $I_{r}^{\star}$ by much.

Claim 4.12. If $\left|\Pi_{1}\right|=o\left(p / r^{4}\right)$, then $\mathbb{E}_{\left(\Pi_{1}, \Sigma, J\right)}\left[\left\|\psi_{r}-\mathcal{D}_{r-1}\right\|\right]=o\left(1 / r^{2}\right)$.

Proof. We have,

$$
\begin{aligned}
\mathbb{E}_{\left(\Pi_{1}, \Sigma, J\right)}\left[\left\|\psi_{r}-\mathcal{D}_{r-1}\right\|\right] & =\mathbb{E}_{\left(\Pi_{1}, \Sigma, J\right)}\left[\left\|\psi_{r}-\operatorname{dist}\left(I_{r}^{\star} \mid(\Sigma, J)\right)\right\|\right] \quad\left(\operatorname{dist}\left(I_{r}^{\star}\right)=\mathcal{D}_{r-1} \text { and } I_{r}^{\star} \perp \Sigma, J\right) \\
& \leq \mathbb{E}_{\left(\Pi_{1}, \Sigma, J\right)}\left[\sqrt{\frac{1}{2} \cdot \mathbb{D}\left(\psi_{r} \| \operatorname{dist}\left(I_{r}^{\star} \mid(\Sigma, J)\right)\right)}\right]
\end{aligned}
$$

(by Pinsker's inequality (Fact 2.8))

$$
\begin{array}{ll}
\leq \sqrt{\frac{1}{2} \cdot \mathbb{E}_{\left(\Pi_{1}, \Sigma, J\right)}\left[\mathbb{D}\left(\psi_{r} \| \operatorname{dist}\left(I_{r}^{\star} \mid(\Sigma, J)\right)\right)\right]} & \text { (by concavity of } \sqrt{\cdot} \text { and Jensen's inequality) } \\
=\sqrt{\frac{1}{2} \cdot \mathbb{I}\left(I_{r}^{\star} ; \Pi_{1} \mid \Sigma, J\right),} \quad \text { (by Fact } 2.7 \text { ) }
\end{array}
$$

which is $o\left(1 / r^{2}\right)$ by Lemma 4.9 .

We are now ready to state the main result of this section. Define the recursive function $e(r):=$ $e(r-1)+o\left(1 / r^{2}\right)($ with $e(0)=0)$. Note that $e(r)=\sum_{i=1}^{r} o\left(1 / i^{2}\right)=o(1)$. We have,

Lemma 4.13. For any $r \geq 1$, any $r$-round protocol $\pi$ for determining $\theta^{\star}$ on $\mathcal{D}_{r}$ with error probability at most $\delta=1 / 3-e(r)$ requires $\Omega\left(p / r^{4}\right)$ communication. 
Proof. We prove this lemma inductively. The base case for $r=1$ follows from Lemma 3.8. Now suppose the result holds for all integers smaller than $r$, and we aim to prove it for the case of $r$-round protocols. Let $\pi$ be a $\delta$-error protocol for estimating $\theta^{\star}$ with $\delta=1 / 3-e(r)$ and assume by contradiction that the communication cost of $\pi$ is $o\left(p / r^{4}\right)$; we use $\pi$ to design a randomized $(r-1)$-round protocol $\pi^{\prime}$ that has communication cost $o\left(p / r^{4}\right)$ and errs w.p. at most $1 / 3-e(r-1)$ on $\mathcal{D}_{r-1}$ and then use averaging argument to fix its randomness to obtain a deterministic protocol that contradicts the induction hypothesis.

Protocol $\pi^{\prime}$ : An $(r-1)$-round protocol for solving instances of $\mathcal{D}_{r-1}$ using protocol $\pi$.

Input: An instance $I \sim \mathcal{D}_{r-1}$. Output: The value of $\theta^{\star}$ in $I$.

(1) Let $N=\left[n_{r}\right]$ and $M=\left[m_{r}\right]$.

(2) Using public randomness, the players sample $\left(\Pi_{1}, \sigma, j^{\star}\right) \sim \mathcal{D}_{r}(N, M)$, i.e., they sample from the joint distribution of the first message of $\pi$ (denoted by $\Pi_{1}$ ), the permutation $\sigma$ over $M$, and the index $j^{\star} \in[p]$.

(3) The players partition $N$ into $k^{2}$ equal-size groups $\mathcal{N}=\left(N_{1}, \ldots, N_{k^{2}}\right)$ (as is done in $\left.\mathcal{D}_{r}\right)$ and the $i$ th player (denoted by $P_{i}$ ) in $I$ mimics the role of the $i$ th player in each group $N_{g} \in \mathcal{N}$ (denoted by $P_{i, g}$ ) individually, as follows:

(a) $P_{i}$ sets the input for $P_{i, g}$ (for $g \in\left[k^{2}\right]$ ) in the instance $I_{i, j^{\star}}$ (in $\mathcal{D}_{r}$ ) as the input of $P_{i}$ in the input instance $I$ mapped via $\sigma$ to $M$ (using the same procedure as in $\mathcal{D}_{r}$ ).

(b) $P_{i}$ samples the input for $P_{i, g}$ (for $g \in\left[k^{2}\right]$ ) in all other instances $I_{i, j}\left(\right.$ for $j \neq j^{\star}$ ) using private randomness from the distribution $I_{i, j} \sim \mathcal{D}_{r} \mid\left(I^{\star}=I, \Pi_{1}, \sigma, j^{\star}\right)$ (we prove this is indeed possible by Proposition 4.14 below).

(4) The players run the protocol $\pi$ on the new sampled instance conditioned on the first message being $\Pi_{1}$, (i.e., run $\pi$ from the second round assuming $\Pi_{1}$ is the content of blackboard after the first round) and output the same answer as $\pi$.

We start by arguing that $\pi^{\prime}$ is indeed a valid protocol; in particular, Line (3b) can be implemented without any communication. To prove that $\pi^{\prime}$ is valid, it suffices to prove the following proposition:

Proposition 4.14. The distribution $\mathcal{I}:=\left(I^{(1)}, \ldots, I^{(n)}\right) \sim\left(\mathcal{D}_{r} \mid I_{r}^{\star}, \Pi_{1}, \Sigma, J\right)$ is a product distribution whereby each $\mathcal{I}_{i}=I^{(i)}$ is sampled from $\mathcal{D}_{r} \mid I_{r}^{\star}(i), \Pi_{1}, \Sigma, J$.

Proof. For any $i \in N$, we prove that $\mathbb{I}\left(\mathcal{I}_{i} ; \mathcal{I}^{-i}, I_{r}^{\star}(-i) \mid I_{r}^{\star}(i), \Pi_{1}, \Sigma, J\right)=0$. By Fact 2.3-(2), this implies that $\mathcal{I}_{i} \perp\left(\mathcal{I}^{-i}, I_{r}^{\star}(-i)\right) \mid I_{r}^{\star}(i), \Pi_{1}, \Sigma$, J, hence proving the proposition. We have,

$$
\begin{aligned}
\mathbb{I}\left(\mathcal{I}_{i} ; \mathcal{I}^{-i}, I_{r}^{\star}(-i) \mid I_{r}^{\star}(i), \Pi_{1}, \Sigma, J\right) & =\mathbb{I}\left(\mathcal{I}_{i} ; \mathcal{I}^{-i}, I_{r}^{\star}(-i) \mid I_{r}^{\star}(i), \Pi_{1, i}, \Pi_{1}^{-i}, \Sigma, J\right) \quad\left(\text { as } \Pi_{1}=\Pi_{1, i}, \Pi_{1}^{-i}\right) \\
& \leq \mathbb{I}\left(\mathcal{I}_{i} ; \mathcal{I}^{-i}, I_{r}^{\star}(-i) \mid I_{r}^{\star}(i), \Pi_{1, i}, \Sigma, J\right),
\end{aligned}
$$

since $\mathcal{I}_{i} \perp \Pi_{1}^{-i} \mid\left(\mathcal{I}^{-i}, I_{r}^{\star}=\left(I_{r}^{\star}(i), I_{r}^{\star}(-i)\right), \Pi_{1, i}, \Sigma, J\right)$ as $\Pi_{1}^{-i}$ is a deterministic function of $\mathcal{I}^{-i}, I_{r}^{\star}, \Sigma, J$, and, hence, we can apply Proposition 2.6.

Furthermore,

$$
\begin{aligned}
\mathbb{I}\left(\mathcal{I}_{i} ; \mathcal{I}^{-i}, I_{r}^{\star}(-i) \mid I_{r}^{\star}(i), \Pi_{1}, \Sigma, J\right) & \leq \mathbb{I}\left(\mathcal{I}_{i} ; \mathcal{I}^{-i}, I_{r}^{\star}(-i) \mid I_{r}^{\star}(i), \Pi_{1, i}, \Sigma, J\right) \\
& \leq \mathbb{I}\left(\mathcal{I}_{i} ; \mathcal{I}^{-i}, I_{r}^{\star}(-i) \mid I_{r}^{\star}(i), \Sigma, J\right),
\end{aligned}
$$

since $\left.\left(\mathcal{I}^{-i}, I_{r}^{\star}(-i)\right) \perp \Pi_{1, i} \mid \mathcal{I}_{i}, I_{r}^{\star}(i), \Sigma, J\right)$ as $\Pi_{1, i}$ is a deterministic function of $I^{(i)}=\left(\mathcal{I}_{i}, I_{r}^{\star}(i)\right), \Sigma, J$ and, hence, we can again apply Proposition 2.6. Finally, $\mathbb{I}\left(\mathcal{I}_{i} ; \mathcal{I}^{-i}, I_{r}^{\star}(-i) \mid I_{r}^{\star}(i), \Sigma, J\right)=0$ by Observation 4.4 and Fact 2.3-(2), implying that $\mathbb{I}\left(\mathcal{I}_{i} ; \mathcal{I}^{-i}, I_{r}^{\star}(-i) \mid I_{r}^{\star}(i), \Pi_{1}, \Sigma, J\right)=0$ as well, proving the proposition. 
It is now easy to see that $\pi^{\prime}$ is indeed an $(r-1)$-round protocol: To sample from the distribution $\mathcal{D}_{r} \mid\left(I^{\star}=I, \Pi_{1}, \Sigma, J\right)$ in Line (3b), each player $i \in N$ needs to sample from the distribution $\mathcal{D}_{r} \mid$ $\left(I_{r}^{\star}(i), \Pi_{1}, \Sigma, J\right)$ (by Proposition 4.14), and this is possible, since $\left(I_{r}^{\star}(i), \Pi_{1}, \Sigma, J\right)$ are all known to $i$. Hence, the players do not need any communication for simulating the first round of protocol $\pi$. We now prove that.

Claim 4.15. $\pi^{\prime}$ is a $\delta^{\prime}$-error protocol for $\mathcal{D}_{r-1}$ for $\delta^{\prime}=1 / 3-e(r-1)$.

Proof. Note that our goal is to calculate the probability that $\pi^{\prime}$ errs given an instance $I \sim \mathcal{D}_{r-1}$. For the sake of analysis, suppose that $I \sim \psi_{r}$ instead, i.e., is sampled from the distribution $\operatorname{dist}\left(I_{r}^{\star} \mid\right.$ $\left.\Pi_{1}, \Sigma, J\right)$ (according to distribution $\mathcal{D}_{r}$ ). In this case, one can see that the distribution of the $r$-round instance constructed by $\pi^{\prime}$ matches the distribution $\mathcal{D}_{r}$. Since $\pi^{\prime}$ outputs the same answer as $\pi$ on this new sampled instance and, since $I=I_{r}^{\star}$ in the new instance, the probability that $\pi^{\prime}$ errs on $\psi_{r}$ is equal to the probability that $\pi$ errs on $\mathcal{D}_{r}$, which in turn is equal to $1 / 3-e(r)$. Now notice that by Claim 4.12, the total variation distance between $\psi_{r}$ and $\mathcal{D}_{r-1}$ is $o\left(1 / r^{2}\right)$ and hence, by Fact 2.9, $\operatorname{Pr}_{\mathcal{D}_{r-1}}\left(\pi^{\prime}\right.$ errs $) \leq \operatorname{Pr}_{\psi_{r}}\left(\pi^{\prime}\right.$ errs $)+o\left(1 / r^{2}\right)=1 / 3-e(r-1)$.

We now formalize the above intuition. Define $R_{\text {pri }}$ and $R_{\text {pub }}$ as, respectively, the private and the public randomness of protocol $\pi^{\prime}$. The probability that $\pi^{\prime}$ errs on an instance $I \sim \mathcal{D}_{r-1}$ can be written as,

$$
\begin{aligned}
& \operatorname{Pr}_{\mathcal{D}_{r-1}}\left(\pi^{\prime} \text { errs }\right)=\mathbb{E}_{I \sim \mathcal{D}_{r-1}} \mathbb{E}_{R_{\text {pub }}}\left[\operatorname{Pr}_{R_{\text {pri }}}\left(\pi^{\prime} \text { errs } \mid R_{\text {pub }}\right)\right] \\
& =\mathbb{E}_{R_{\text {pub }}} \mathbb{E}_{I \sim \mathcal{D}_{r-1} \mid R_{\text {pub }}}\left[\operatorname{Pr}_{R_{\text {pri }}}\left(\pi^{\prime} \text { errs } \mid R_{\text {pub }}\right)\right] \\
& \left.=\mathbb{E}_{\left(\Pi_{1}, \Sigma, J\right)} \mathbb{E}_{I \sim \mathcal{D}_{r-1} \mid\left(\Pi_{1}, \Sigma, J\right)}\left[\operatorname{Pr}_{R_{\text {pri }}}\left(\pi^{\prime} \text { errs } \mid \Pi_{1}, \Sigma, J\right)\right] \quad \text { (as } R_{\text {pub }}=\left(\Pi_{1}, \Sigma, J\right)\right) \\
& \leq \mathbb{E}_{\left(\Pi_{1}, \Sigma, J\right)}\left[\mathbb{E}_{I \sim \psi_{i} \mid\left(\Pi_{1}, \Sigma, J\right)}\left[\operatorname{Pr}_{R_{\mathrm{pri}}}\left(\pi^{\prime} \operatorname{errs} \mid \Pi_{1}, \Sigma, J\right)\right]+\left\|\mathcal{D}_{r-1}-\left(\psi_{i} \mid\left(\Pi_{1}, \Sigma, J\right)\right)\right\|\right] \\
& \text { (by Fact 2.9) } \\
& =\mathbb{E}_{\left(\Pi_{1}, \Sigma, J\right)}\left[\mathbb{E}_{I \sim \psi_{i} \mid\left(\Pi_{1}, \Sigma, J\right)}\left[\operatorname{Pr}_{R_{\mathrm{pri}}}\left(\pi^{\prime} \operatorname{errs} \mid \Pi_{1}, \Sigma, J\right)\right]\right]+\mathbb{E}\left[\left\|\mathcal{D}_{r-1}-\psi_{i}\right\|\right] \\
& =\mathbb{E}_{\left(\Pi_{1}, \Sigma, J\right)}\left[\mathbb{E}_{I \sim \psi_{i} \mid\left(\Pi_{1}, \Sigma, J\right)}\left[\operatorname{Pr}_{R_{\text {pri }}}\left(\pi^{\prime} \operatorname{errs} \mid \Pi_{1}, \Sigma, J\right)\right]\right]+o\left(1 / r^{2}\right) \\
& =\mathbb{E}_{\left(\Pi_{1}, \Sigma, J\right)}\left[\mathbb{E}_{I \sim \psi_{i} \mid\left(\Pi_{1}, \Sigma, J\right)}\left[\operatorname{Pr}_{\mathcal{D}_{r}}\left(\pi^{\prime} \operatorname{errs} \mid I_{r}^{\star}=I, \Pi_{1}, \Sigma, J\right)\right]\right]+o\left(1 / r^{2}\right) \\
& \text { (the distribution of sampled instances in } \pi^{\prime}\left(\text { via } R_{\text {pri }} \text { ) matches } \mathcal{D}_{r} \mid I^{\star}=I, \Pi_{1}, \Sigma, J\right) \\
& =\mathbb{E}_{\left(\Pi_{1}, \Sigma, J\right)}\left[\mathbb{E}_{I \sim \psi_{i} \mid\left(\Pi_{1}, \Sigma, J\right)}\left[\operatorname{Pr}_{\mathcal{D}_{r}}\left(\pi \operatorname{errs} \mid I_{r}^{\star}=I, \Pi_{1}, \Sigma, J\right)\right]\right]+o\left(1 / r^{2}\right) \\
& \text { (the output of } \pi^{\prime} \text { and } \pi \text { is the same) } \\
& =\mathbb{E}_{\left(I_{r}^{\star}, \Pi_{1}, \Sigma, J\right)}\left[\operatorname{Pr}_{\mathcal{D}_{r}}\left(\pi \operatorname{errs} \mid I_{r}^{\star}, \Pi_{1}, \Sigma, J\right)\right]+o\left(1 / r^{2}\right) \quad\left(\psi_{i}=\operatorname{dist}\left(I_{r}^{\star} \mid \Pi_{1}, \Sigma, J\right) \text { by definition }\right) \\
& =\operatorname{Pr}_{\mathcal{D}_{r}}(\pi \text { errs })+o\left(1 / r^{2}\right)=1 / 3-e(r)+o\left(1 / r^{2}\right)=1 / 3-e(r-1),
\end{aligned}
$$

finalizing the proof.

Lemma 4.13 now follows from Claim 4.15 by an averaging argument, since we can fix the randomness in $\pi^{\prime}$ to obtain a deterministic protocol $\pi^{\prime \prime}$ that uses $o\left(p / r^{4}\right)$ bits of communication and errs w.p. at most $1 / 3-e(r-1)$ on $\mathcal{D}_{r-1}$, a contradiction with the induction hypothesis.

Theorem 3 now easily follows from Lemma 4.13.

Proof of TheOrem 4.1. Let $\pi$ be a $\left(\frac{1}{r} \cdot m^{\frac{1-2 \varepsilon}{2 r+1}}\right)$-approximation, (1/4)-error protocol for subadditive combinatorial auctions on the distribution $\mathcal{D}_{r}$. By Claim $4.8, \pi$ is also a $(1 / 4+o(1))$ error protocol for $\theta^{\star}$ estimation on $\mathcal{D}_{r}$. Since $(1 / 4+o(1))<1 / 3-e(r)$, by Lemma 4.13 , we have $\|\pi\|=\Omega\left(p / r^{4}\right)=\exp \left(\Theta\left(k^{\varepsilon}\right)\right) / r^{4}=\exp \left(m^{\Omega(\varepsilon / r)}\right) / r^{4}=\exp \left(m^{\Omega(\varepsilon / r)}\right)$, as $k=m^{\Omega(1 / r)}$ and $r=$ $o\left(\frac{\log m}{\log \log m}\right)$. Re-parametrizing $\varepsilon$ by $\varepsilon / 2$ in the lower bound argument finalizes the proof. 


\section{CONCLUSION}

In this article, we studied the role of interaction in obtaining efficient allocations in subadditive combinatorial auctions. We showed that for any $r \geq 1$, any $r$-round protocol that uses polynomial communication can only achieve an $\Omega\left(\frac{1}{r} \cdot m^{1 /(2 r+1)}\right)$ approximation to the social welfare. This settles an open question posed in References $[2,13]$ on the round-approximation tradeoff of polynomial communication protocols for subadditive combinatorial auctions.

An immediate corollary of our main result is that $\Omega\left(\frac{\log m}{\log \log m}\right)$ rounds of interaction are necessary for obtaining an efficient allocation in subadditive combinatorial auctions. The qualitative message of this theoretical result is that not only interaction between individuals in a market is necessary for obtaining an efficient allocation; one indeed needs a modest amount of interaction (growing with the market size) to obtain an efficient allocation. This further supports the point of view of Reference [13] on the necessity of interaction for economic efficiency.

An interesting direction for future research, also advocated by Reference [13], is to consider the case of bidders with submodular valuations. It is known that obtaining a better than $(1-1 / 2 e)$ approximation to social welfare in submodular combinatorial auctions requires exponential communication [16] (regardless of the number of rounds of interaction). However, no better lower bounds are known for bounded-round protocols (even for simultaneous ones). Another very interesting direction is to understand the communication complexity of subadditive combinatorial auctions when the numbers of players and rounds are only small constants-say, two players and only one round of communication. This problem is highly motivated due to its relevance to understanding the gap between the power of truthful and non-truthful mechanisms for this problem by a characterization result due to Dobzinski [12]; see also Reference [8] for more details on this connection and also tight bounds for simultaneous two-player combinatorial auctions with XOS valuations. We remark that our techniques in this article were heavily tailored toward markets with many players and extending them to the case of constantly many players seems quite nontrivial and interesting.

\section{ACKNOWLEDGMENTS}

I thank my advisor Sanjeev Khanna for many helpful advice and comments and Jamie Morgenstern for helpful discussions in the earlier stages of this work. I am also grateful to Rotem Oshman for helpful conversations on the results in Reference [9] and to Matthew Weinberg for bringing Reference [8] to my attention. Finally, I would like to thank the anonymous reviewers of EC and TEAC for many insightful comments and suggestions.

\section{REFERENCES}

[1] Ron Aharoni, Paul Erdös, and Nathan Linial. 1988. Optima of dual integer linear programs. Combinatorica 8, 1 (1988), 13-20.

[2] Noga Alon, Noam Nisan, Ran Raz, and Omri Weinstein. 2015. Welfare maximization with limited interaction. In Proceedings of the IEEE 56th Symposium on Foundations of Computer Science (FOCS'15). 1499-1512.

[3] Sepehr Assadi. 2017. Combinatorial auctions do need modest interaction. In Proceedings of the ACM Conference on Economics and Computation (EC'17). 145-162.

[4] Sepehr Assadi and Sanjeev Khanna. 2018. Tight bounds on the round complexity of the distributed maximum coverage problem. In Proceedings of the 29th ACM-SIAM Symposium on Discrete Algorithms (SODA'18). 2412-2431.

[5] Sepehr Assadi, Sanjeev Khanna, and Yang Li. 2017. On estimating maximum matching size in graph streams. In Proceedings of the 28th ACM-SIAM Symposium on Discrete Algorithms (SODA'17). 1723-1742.

[6] Ashwinkumar Badanidiyuru, Shahar Dobzinski, Hu Fu, Robert Kleinberg, Noam Nisan, and Tim Roughgarden. 2012. Sketching valuation functions. In Proceedings of the 23rd ACM-SIAM Symposium on Discrete Algorithms (SODA'12). $1025-1035$.

[7] Maria-Florina Balcan, Florin Constantin, Satoru Iwata, and Lei Wang. 2012. Learning valuation functions. In Proceedings of the 25th Conference on Learning Theory (COLT'12). 4.1-4.24. 
[8] Mark Braverman, Jieming Mao, and S. Matthew Weinberg. 2018. On simultaneous two-player combinatorial auctions. In Proceedings of the 29th ACM-SIAM Symposium on Discrete Algorithms (SODA'18). 2256-2273.

[9] Mark Braverman and Rotem Oshman. 2017. A rounds vs. communication tradeoff for multi-party set disjointness. In Proceedings of the 58th IEEE Symposium on Foundations of Computer Science (FOCS'17). 144-155.

[10] Patrick Briest, Piotr Krysta, and Berthold Vöcking. 2011. Approximation techniques for utilitarian mechanism design. SIAM F. Comput. 40, 6 (2011), 1587-1622.

[11] Thomas M. Cover and Joy A. Thomas. 2006. Elements of Information Theory (2nd ed.). Wiley.

[12] Shahar Dobzinski. 2016. Computational efficiency requires simple taxation. In Proceedings of the IEEE 57th Symposium on Foundations of Computer Science (FOCS'16). 209-218.

[13] Shahar Dobzinski, Noam Nisan, and Sigal Oren. 2014. Economic efficiency requires interaction. In Proceedings of the Symposium on Theory of Computing (STOC'14). 233-242.

[14] Shahar Dobzinski, Noam Nisan, and Michael Schapira. 2005. Approximation algorithms for combinatorial auctions with complement-free bidders. In Proceedings of the 37th ACM Symposium on Theory of Computing. 610-618.

[15] Shahar Dobzinski and Michael Schapira. 2006. An improved approximation algorithm for combinatorial auctions with submodular bidders. In Proceedings of the 17th ACM-SIAM Symposium on Discrete Algorithms (SODA'06). 1064-1073.

[16] Shahar Dobzinski and Jan Vondrák. 2013. Communication complexity of combinatorial auctions with submodular valuations. In Proceedings of the 24th ACM-SIAM Symposium on Discrete Algorithms (SODA'13). 1205-1215.

[17] Devdatt P. Dubhashi and Alessandro Panconesi. 2009. Concentration of Measure for the Analysis of Randomized Algorithms. Cambridge University Press.

[18] Paul Duetting, Michal Feldman, Thomas Kesselheim, and Brendan Lucier. 2017. Prophet inequalities made easy: Stochastic optimization by pricing non-stochastic inputs. In Proceedings of the 58th IEEE Symposium on Foundations of Computer Science (FOCS'17). 540-551.

[19] Paul Dütting and Thomas Kesselheim. 2017. Best-response dynamics in combinatorial auctions with item bidding. In Proceedings of the 28th ACM-SIAM Symposium on Discrete Algorithms (SODA'17). 521-533.

[20] Uriel Feige. 2009. On maximizing welfare when utility functions are subadditive. SIAM J. Comput. 39, 1 (2009), 122142.

[21] Uriel Feige and Jan Vondrák. 2006. Approximation algorithms for allocation problems: Improving the factor of 1-1/e. In Proceedings of the 47th IEEE Symposium on Foundations of Computer Science (FOCS'06). 667-676.

[22] Michal Feldman, Hu Fu, Nick Gravin, and Brendan Lucier. 2013. Simultaneous auctions are (almost) efficient. In Proceedings of the Symposium on Theory of Computing Conference (STOC'13). 201-210.

[23] Michal Feldman, Nick Gravin, and Brendan Lucier. 2015. Combinatorial auctions via posted prices. In Proceedings of the 26th ACM-SIAM Symposium on Discrete Algorithms (SODA'15). 123-135.

[24] Russell Impagliazzo and Valentine Kabanets. 2010. Constructive proofs of concentration bounds. In Proceedings of the 13th International Workshop on Approximation, Randomization, and Combinatorial Optimization (APPROX'10) and 14th International Workshop on Randomization and Approximation Techniques in Computer Science (RANDOM'10). 617-631.

[25] Ron Lavi and Chaitanya Swamy. 2011. Truthful and near-optimal mechanism design via linear programming. F. ACM 58, 6 (2011), 25:1-25:24.

[26] Benny Lehmann, Daniel J. Lehmann, and Noam Nisan. 2006. Combinatorial auctions with decreasing marginal utilities. Games Econ. Behav. 55, 2 (2006), 270-296.

[27] László Lovász. 1975. On the ratio of optimal integral and fractional covers. Disc. Math. 13, 4 (1975), 383-390.

[28] Peter Bro Miltersen, Noam Nisan, Shmuel Safra, and Avi Wigderson. 1998. On data structures and asymmetric communication complexity. F. Comput. Syst. Sci. 57, 1 (1998), 37-49.

[29] Noam Nisan. 2002. The communication complexity of approximate set packing and covering. In Proceedings of the 29th International Colloquium on Automata, Languages and Programming (ICALP'02). 868-875.

[30] Noam Nisan and Ilya Segal. 2006. The communication requirements of efficient allocations and supporting prices. f. Econ. Theor. 129, 1 (2006), 192-224.

[31] Alessandro Panconesi and Aravind Srinivasan. 1997. Randomized distributed edge coloring via an extension of the Chernoff-Hoeffding bounds. SIAM F. Comput. 26, 2 (1997), 350-368.

[32] Pranab Sen and Srinivasan Venkatesh. 2008. Lower bounds for predecessor searching in the cell probe model. f. Comput. Syst. Sci. 74, 3 (2008), 364-385.

[33] Jan Vondrák. 2008. Optimal approximation for the submodular welfare problem in the value oracle model. In Proceedings of the 40th ACM Symposium on Theory of Computing. 67-74.

[34] Andrew Chi-Chih Yao. 1979. Some complexity questions related to distributive computing (preliminary report). In Proceedings of the 11th ACM Symposium on Theory of Computing. 209-213.

Received November 2017; revised September 2018; accepted July 2019 